\title{
On-line Appendix: Generalized Correlation and Kernel Causality with Applications in Development Economics
}

\author{
Hrishikesh D. Vinod* \\ first version, July 14, 2015
}

\begin{abstract}
This document describes on-line supplementary material explaining the data and $\mathrm{R}$ software to accompany my paper Vinod (2015) appearing in Communications in Statistics - Simulation and Computation. The aim of this supplement is to assist the reader in extending and implementing the ideas in my paper. Included $\mathrm{R}$ code can be used as a template allowing one to use my methods on any data of interest to the reader. My software here is not claimed to be efficient, but does work. Explanations of software steps are often included, but not claimed to be complete. Hence some familiarity with $\mathrm{R}$ is needed to use it properly. The software tools and their descriptions provided here need additional work to bring them up to the standard of an official $R$ package.
\end{abstract}

\section{Introduction}

This document is intended to be read in conjunction with my paper Vinod (2015) published in Communications in Statistics - Simulation and Computation (CSSC). This document is focused only on implementation and data

*Vinod: Professor of Economics, Fordham University, Bronx, New York, USA 10458. E-mail: vinod@fordham.edu. I thank Shurong Zheng for giving me the R code for GMC. A version of the original paper (of which this is a supplement) was a keynote address to the 50th annual (Golden Jubilee) meeting of the Indian Econometric Society in Mumbai, December 2013. I thank two referees for several detailed and helpful suggestions. 
analysis, and will not repeat the explained theory from the paper. Most $\mathrm{R}$ code snippets are named for cross-reference purposes. The names always start with "R-".

There is considerable $\mathrm{R}$ code provided here which can be developed into an $\mathrm{R}$ package. At the minimum the code can serve as a template for specific graphics or computational applications with minor modifications. The code is seen in red font ready to be copied and pasted by the user. All commands of the type 'require()' or 'library()' will not work unless the particular $\mathrm{R}$ package named in parentheses is first loaded at the $\mathrm{R}$ installation where the code is used.

The blue font is reserved for outputs from the code. The outputs of many code snippets is suppressed and /or abridged for brevity.

\section{$2 \quad \mathrm{R}$ software Initialization}

While many $\mathrm{R}$ experts do not agree with me, I prefer to replace the $\mathrm{R}$ prompt $(>)$ implicit in the $\mathrm{R}$ software with one space and the $\mathrm{R}$ continuation symbol $(+)$ with two spaces. This change permits direct copy and paste from this file into any user's R console without modifications, avoiding confusion with the greater than and plus symbols also used in R. I also prefer to use the one stroke (=) symbol for assignment rather than $(<-)$ requiring 4 strokes after including spaces commonly used in $\mathrm{R}$.

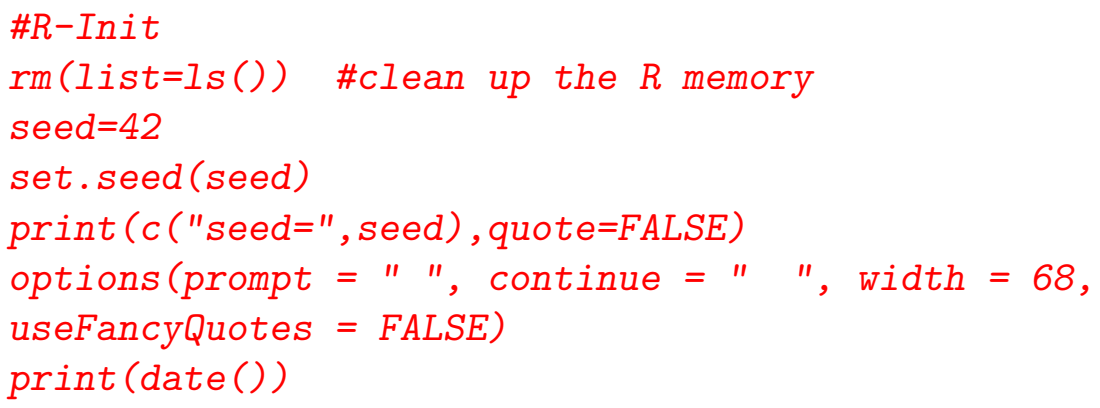

It is recommended that the reader copy and paste this code named " $\mathrm{R}$ Init" before each new project. The next section describes our data sources before use in the subsequent sections. 


\section{Details Regarding the Nine Variables in Economic Development (EcDev) Data}

Our data are publicly available at the UN, (See http://hdr.undp.org/en/ data), the World Bank http://search.worldbank.org/data, etc. The supplementary material to this paper allows the reader to reproduce our results. Some data and country codes follow the Association of Religion Data Archives (ARDA) www. TheARDA.com, with the Principal Investigator Jaime Harris.

1. GRO: the least squares annual growth rate in Gross Domestic Product (GDP) used with constant GDP per capita data in local currency units. Sample growth rates are China $=7.9$, India $=3.6$, Mexico $=1.7$ and $\mathrm{USA}=1.9$.

2. INHDI: Inequality-adjusted Human Development Index.

This measure adjusts a country's HDI score based on its scores on three additional indexes: the inequality-adjusted life expectancy index, the inequality-adjusted education index and the inequality-adjusted income index. Sample scores are Albania $=0.627$, Bangladesh $=0.321$, India $=0.365, \mathrm{USA}=0.799$ and Pakistan $=0.336$.

3. GINI: Income Gini coefficient. This is a measure of the deviation of the distribution of income (or consumption) among individuals or households within a country from a perfectly equal distribution. A Lorenz curve plots the cumulative percentages of total income received against the cumulative number of recipients, starting with the poorest individual or household. The Gini index measures the area between the Lorenz curve and a hypothetical line of absolute equality, expressed as a percentage of the maximum area under the line. A value of zero represents absolute equality, a value of 100 absolute inequality. Sample scores are Albania $=33$, Bangladesh $=31$, India $=36.8$, Iran $=38.5$ and $\mathrm{USA}=40.6$.

4. GII: A country's score on the Gender Inequality Index (GII), a composite index measuring loss in achievements in three dimensions of human development: reproductive health (measured by the maternal mortality ratio and the adolescent fertility rate), empowerment (measured by the

female and male population with at least a secondary education and by 
the female and male shares of parliamentary seats) and the labor market due to inequality between genders (measured by female and male labor force participation rates). These scores are high in countries with large Muslim populations as: Egypt $=0.714$ and India $=0.748$ and low in Westernized countries as Japan $=0.273$ and The Netherlands $=0.174$. The USA $=0.400$ is somewhat higher than expected.

5. MPI: Multidimensional Poverty Index. This indicates the share of the population that is multidimensionally poor adjusted by the intensity of the deprivations. These deprivation data are not reported for rich countries. The scores are Albania $=0.004$, Bangladesh $=0.291$, India $=0.296$ and Pakistan $=0.275$. India is among countries showing the worst deprivations for her poor.

6. GEI: Governance Effectiveness Index from the World Bank ranging between -2.5 to 2.5. For examples: Australia=1.74, India $=(-0.03)$, Pakistan $=(-0.82), \mathrm{UK}=1.55$, Japan $=1.35$. It reflects perceptions of the quality of public services, the quality of the civil service and the degree of its independence from political pressures, the quality of policy formulation and implementation, and the credibility of the government's commitment to such policies. The data are available for 1996-2011 for over 200 countries. We use the 2011 data.

7. Entp: Entrepreneurship index is what the World Bank researchers call "New Business Density". A high density indicates prevalence of entrepreneurship in a country, calculated as the number of newly registered limited liability companies per 1,000 working-age people (those ages 15-64), World Bank (2013). For examples: Australia=6.17, India $=0.09$, Pakistan $=0.03, \mathrm{UK}=8.32$, Japan $=1.1$.

8. ECI: Economic Complexity Index developed at MIT and explained in Hidalgo and Hausman (2009). The authors claim that the greater the number of complex exchanges in international trade of goods undertaken (manufactured) in a country, the higher is its economic growth potential. For examples: Brazil $=0.244$, India $=0.247$, Pakistan $=(-$ 0.398), Philippines $=0.032, \mathrm{UK}=1.558$, Japan $=2.316$, reflecting that Pakistan does not do much of complex manufacturing and Philippines does more than Pakistan. 
9. AidPC: Per Capita net official development assistance (ODA) consists of disbursements of loans made on concessional terms (net of repayments of principal) and grants by official agencies of the members of the Development Assistance Committee (DAC), by multilateral institutions, and by non-DAC countries to promote economic development and welfare. One divides by the midyear population estimate. It includes loans with a grant element of at least 25 percent (calculated at a rate of discount of 10 percent). Data are available online at: www.oecd.org/dac/stats/idsonline. World Bank population estimates are used for the denominator. For examples: Pakistan=20, India $=3$, Iraq $=60$, Bhutan $=197$, Kenya $=59$.

\section{$4 \quad \mathrm{R}$ software for reading Economic Development (EcDev) Data}

We use the country codes by ARDA available at http://www.fordham.edu/ economics/vinod/ARDAcountryCodes.xls. The data are available in $\mathrm{R}$ format at: http://www . fordham.edu/economics/vinod/DevEcData.Rdata. If the Rdata file does not work an alternative version 'DevEc10.csv' a comma separated csv file is also available at the same Internet location. Its use is shown below.

The data for 250 countries contains lots of missing data (NAs). The code named "R-get-EcDev-data" loads entire data into user's R console for the chosen nine variables (abbreviations listed in the previous section) and country code.

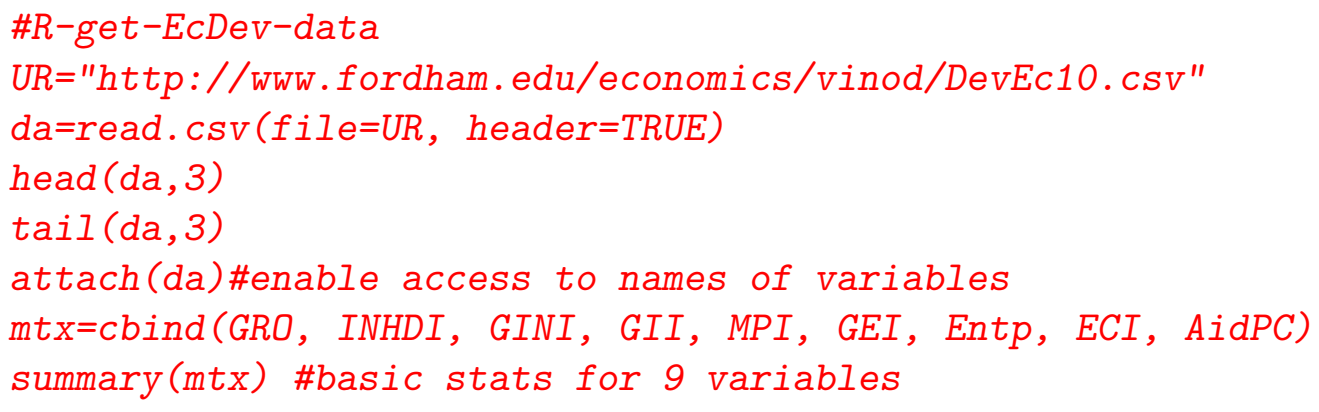

The output from the above code allows one to briefly view the data by reporting the data for the first and last three countries only. 


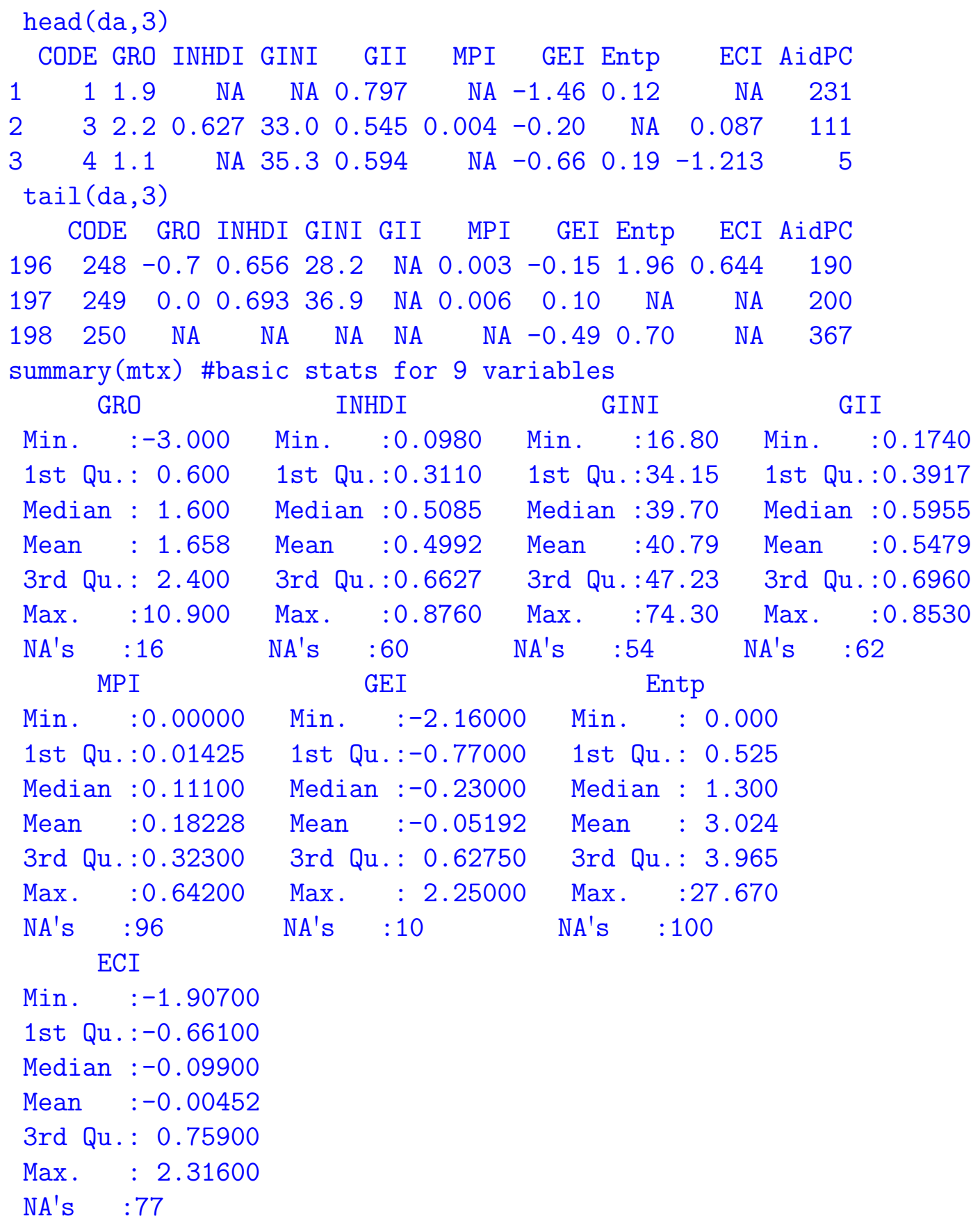

The next task is to compute the usual correlation coefficients. I recommend using the package 'Hmisc' for this purpose. The output of the code 
below named "R-plot-EcDev" consists of two figures. It is suppressed here for brevity. The 'corrgram' is printed in the journal article as "Figure 1: Corrogram for nine economic development variables."

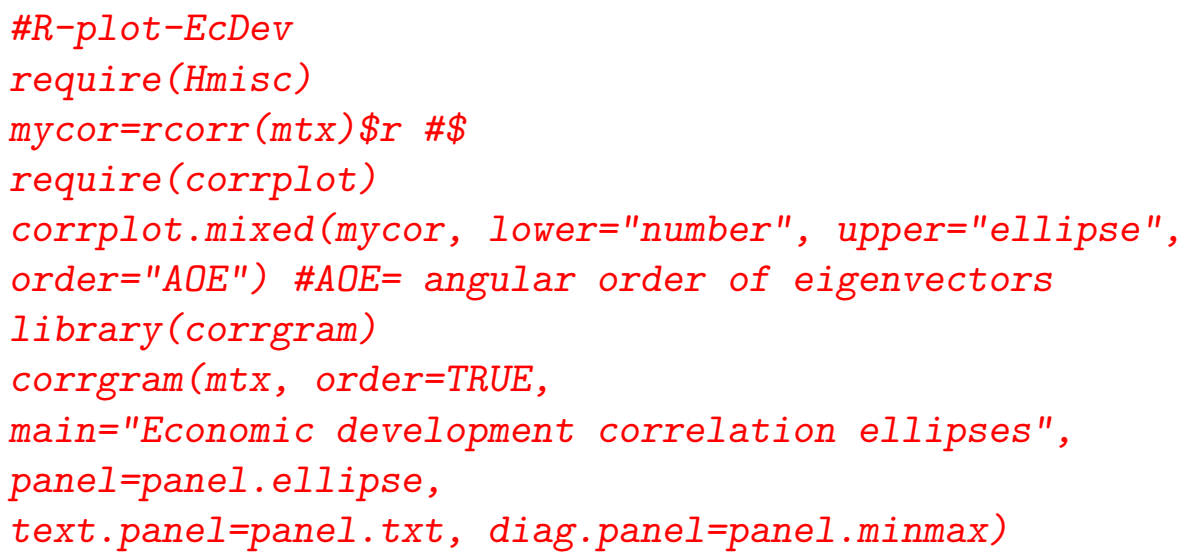

\section{$5 \quad$ Plotting Bivariate Density for EcDev data}

Now we provide the $\mathrm{R}$ code for plotting Bivariate density seen as "Figure 2: Joint density between economic growth (GRO) and income in- equality (GINI)" in my CSSC paper.

One needs to do pair-wise deletion of missing data or NA's by using the code named "R-napair" providing my function called 'napair.'

The code named "R-napair-Dev-data" further below uses 'napair' function on EcDev data. It renames the pair-wise matched data as GRO2 and GINI2, while keeping the original GRO and GINI data intact, since the original data will have to be matched eventually with seven other variables.

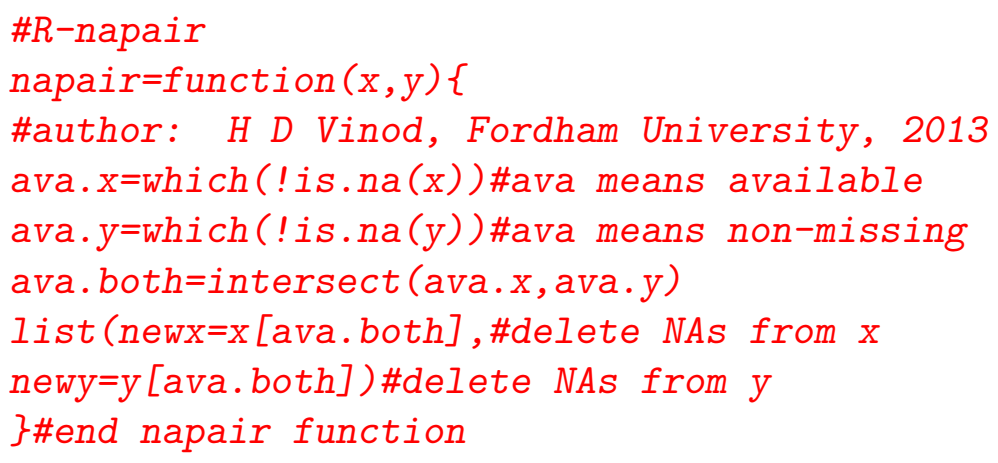




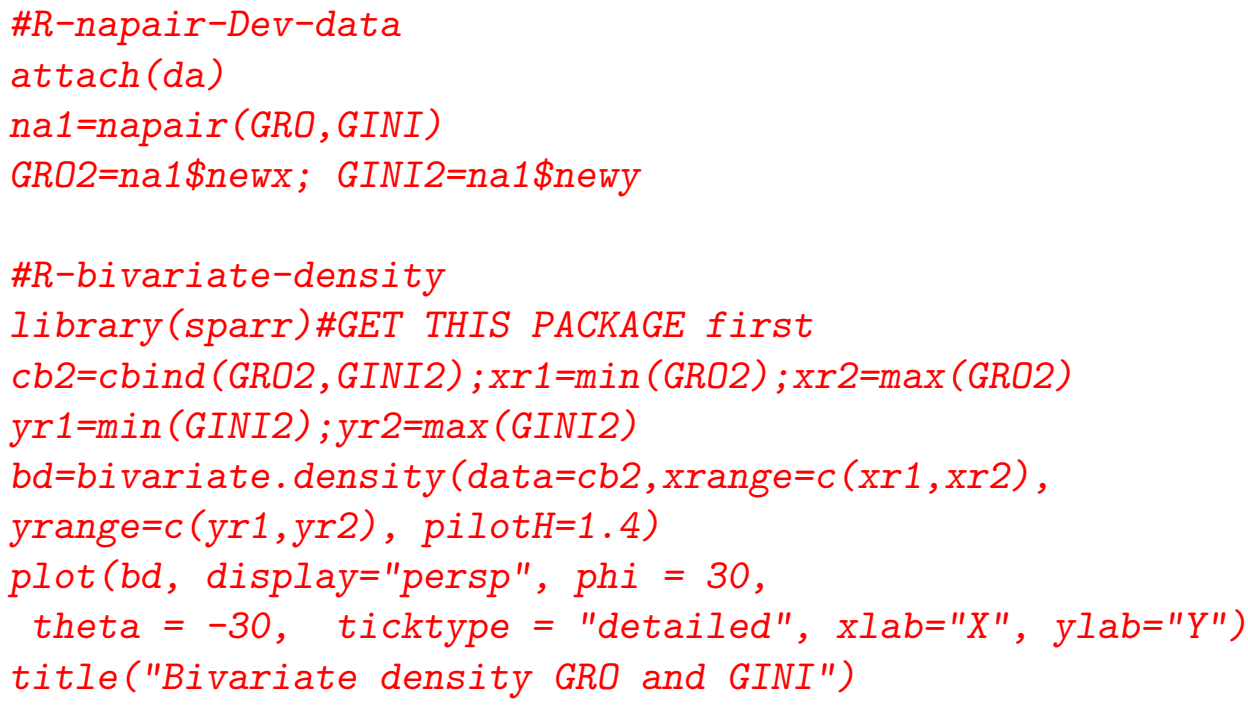

The code named "R-bivariate-density" above does the actual plotting of the data after NAs are removed and series are matched.

\section{Computing Generalized Correlation Matrix}

The code named "R-gmcxy.np-function" below is my R function which in turn relies on the 'np' package to do kernel regressions in equations (1) and (2) in my CSSC paper. The function 'npregbw' suitably sets up the bandwidths.

The code named "R-gmcmtx0-function" provides my $\mathrm{R}$ function to compute the $R^{*}$ (new asymmetric generalized correlation matrix) described in Section 2.5 and defined in equation (10) of my CSSC paper. The zero in the name 'gmcmtx0' is intended to distinguish it from a more CPU-timeconsuming bootstrap version 'gmcmtx,' described later.

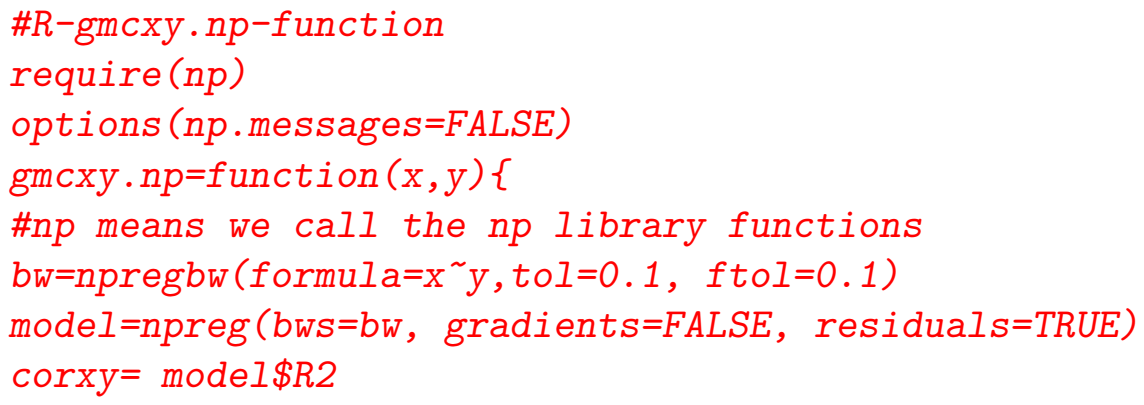




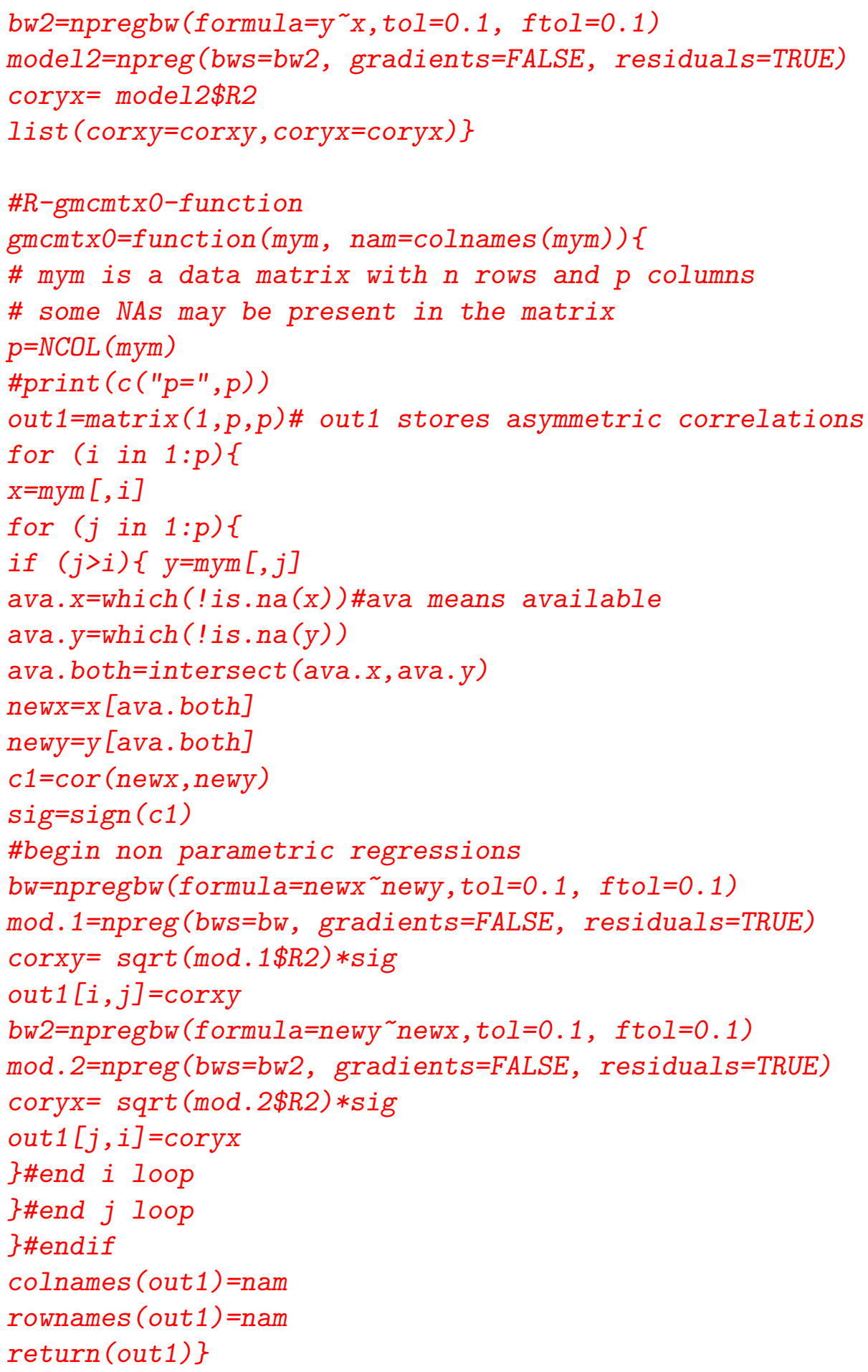


Now we are ready to use the function 'gmcmtx0' on any data having $T$ observations on $p$ variables in columns, organized in the form of a $T \times p$ matrix.

Our first example is the European crime data from Section 3.3 of my CSSC paper.

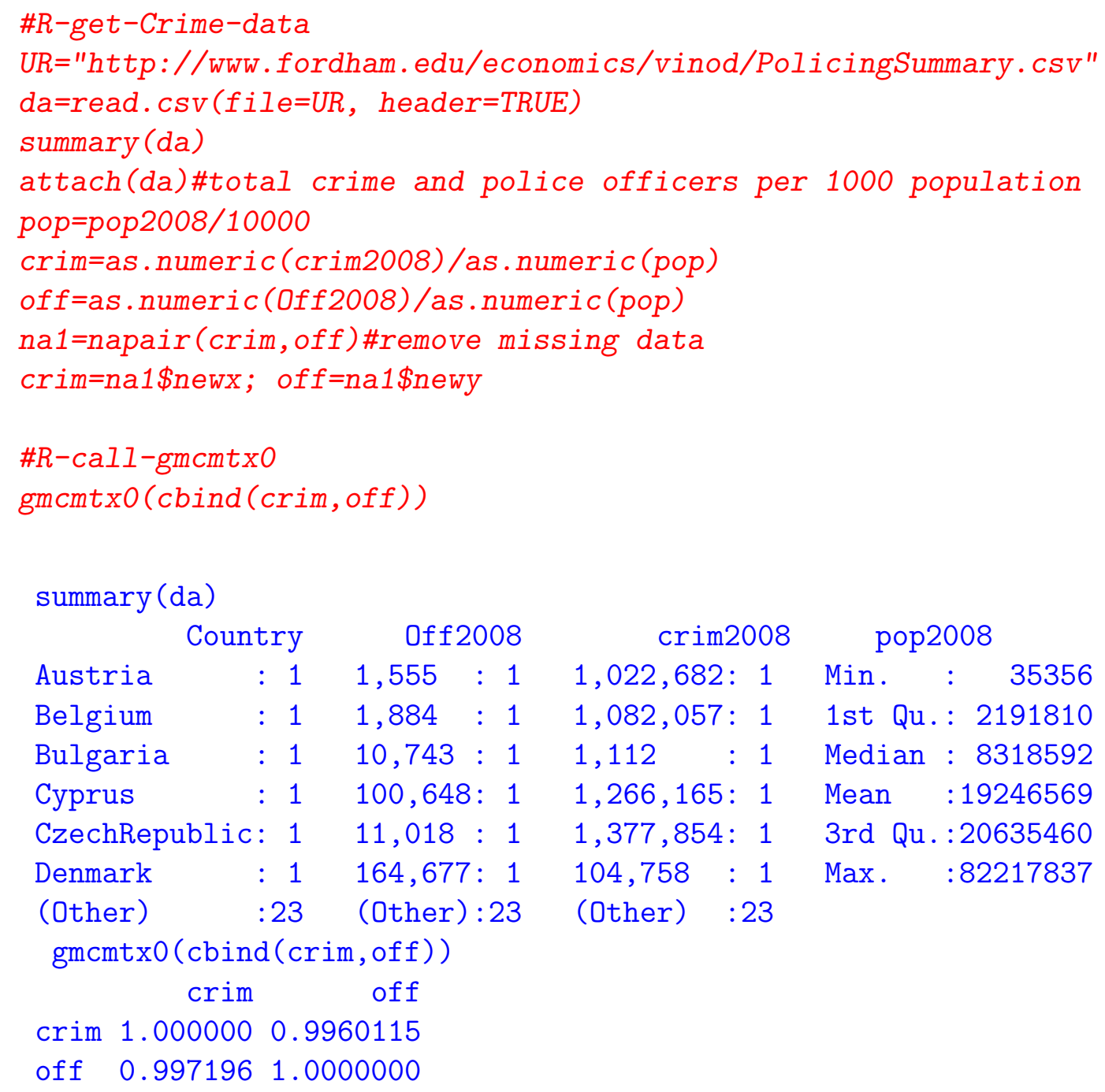

Note that row variable $X_{i}$ is the "effect" and the column variable $X_{j}$ is the predictor or the "cause." The value of $R^{*}$ in the column for crime is slightly larger than the value in the off column for police officers. It stands to reason that high crime causes larger police deployment. 
Now consider the Internet advertising data of Section 3.4 of my CSSC paper. The code named "R-read-ad- $R^{*}$ " reads in the Internet advertising data and reports the $R^{*}$ matrix computed by using the function 'gmcmtx0' given above.

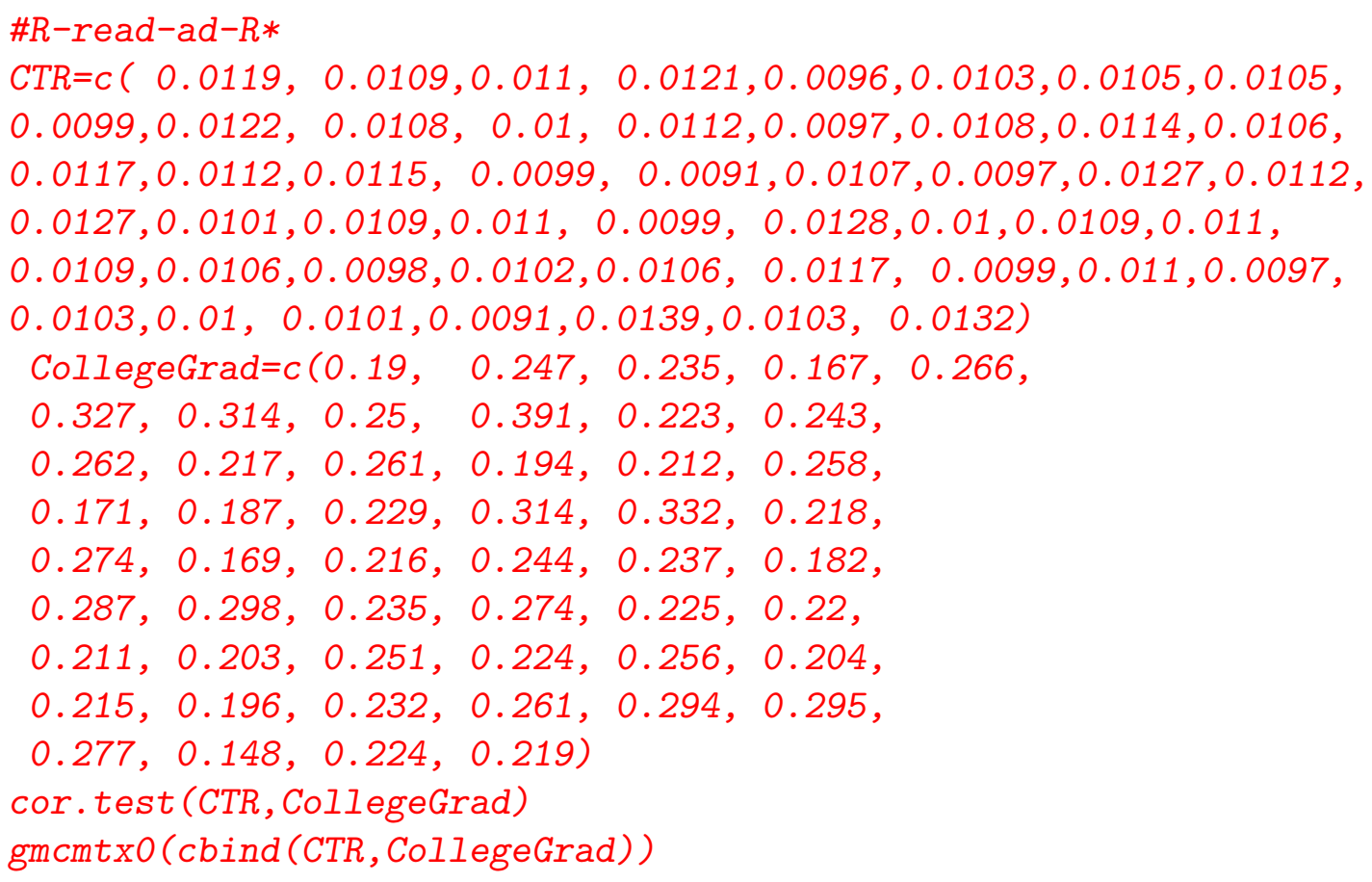

The output of the command 'cor.test' is included first. It includes the t-test of the Pearson correlation coefficient, a confidence interval and p-value. It is followed by the estimated $R^{*}$ matrix for the Internet advertising data.

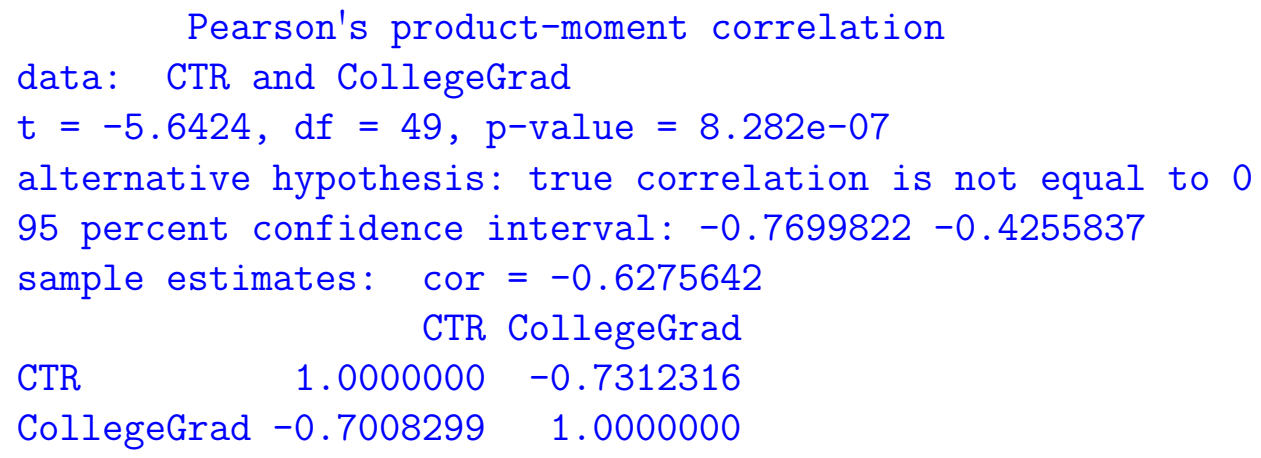


Again, the row variable $X_{i}$ is the "effect" and the column variable $X_{j}$ is the "cause." The magnitude 0.73 exceeds 0.70 suggesting that college graduates are not easy customers of Internet advertising.

Consider the short data for 7 regions on housing bubble in Section 3.5 of my CSSC paper which can be manually entered.

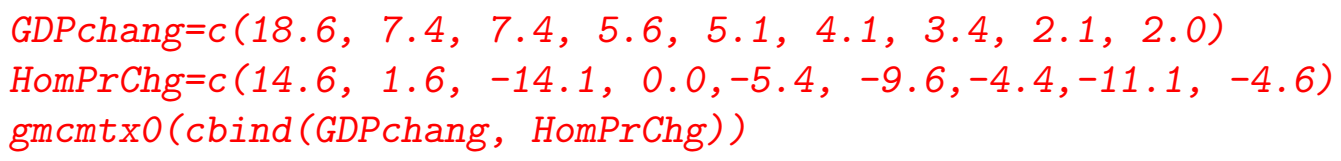

The output $R^{*}$ matrix is given

$$
\text { GDPchang HomPrChg }
$$

GDPchang $1.0000000 \quad 0.968136$

HomPrChg $0.7838597 \quad 1.000000$

Since the row variable $X_{i}$ is the "effect" and the column variable $X_{j}$ is the cause," HomPrChg has a stronger effect (0.968) on GDPchang than vice versa. This supports the notion that the housing bubbles during the particular time period may have hurt the growth in GDP.

Using the nine variables from 'EcDev' data in the matrix called ' $m$ tx' above we can get the matrix of $r^{*}\left(X_{i} \mid X_{j}\right)$ defined by eq. (10) in the text of my CSSC paper by the following command. The generalized correlation matrix does not appear in Section 4 of the CSSC paper.

$r s=g m c m t x O(m t x)$

print (rs, digits=2)

The output given below is abridged to only two digits to fit the width of the page. The actual $R^{*}$ matrix in the memory of $\mathrm{R}$ has more than eight digits than what is displayed here. Tables 5 and 6 of my CSSC paper displays additional digits for 36 unique pairs and explicitly identify the "cause" by name.

\begin{tabular}{|c|c|c|c|c|c|c|c|c|c|}
\hline & GRO & INHDI & GINI & GII & MPI & GEI & Entp & ECI & AidPC \\
\hline GRO & 1.000 & 0.370 & -0.061 & -0.29 & -0.28 & 0.380 & 0.317 & 0.40 & -0 . \\
\hline INHDI & 0.385 & 1.000 & -0.589 & -0.94 & -0.94 & 0.863 & 0.736 & 0.83 & -0.511 \\
\hline GINI & -0.049 & -0.566 & 1.000 & 0.68 & 0.62 & -0.486 & 0.058 & -0.53 & 0.134 \\
\hline
\end{tabular}




$\begin{array}{lrrrrrrrrr}\text { GII } & -0.307 & -0.954 & 0.682 & 1.00 & 0.83 & -0.864 & -0.746 & -0.86 & 0.593 \\ \text { MPI } & -0.271 & -0.956 & 0.289 & 0.82 & 1.00 & -0.641 & -0.613 & -0.71 & 0.445 \\ \text { GEI } & 0.500 & 0.889 & -0.436 & -0.87 & -0.62 & 1.000 & 0.733 & 0.81 & -0.315 \\ \text { Entp } & 0.306 & 0.431 & 0.060 & -0.46 & -0.63 & 0.507 & 1.000 & 0.25 & 0.166 \\ \text { ECI } & 0.406 & 0.843 & -0.557 & -0.83 & -0.70 & 0.811 & 0.514 & 1.00 & -0.285 \\ \text { AidPC } & -0.074 & -0.044 & 0.076 & 0.14 & 0.42 & -0.044 & 0.919 & -0.39 & 1.000\end{array}$

\section{Software for Multivariate Tools for Ameliorating Misspecifications}

This section provides software for Section 2.5 of my CSSC paper. It begins with the $R^{*}$ matrix and computes its minors and cofactors, requiring $\mathrm{R}$ functions for those tasks. The minors of a matrix are given by my $\mathrm{R}$ function called 'minor' after removing row $\mathrm{r}$ and column c. The cofactors are signed minors and are given by the function called 'cofactor'. Both are given next.

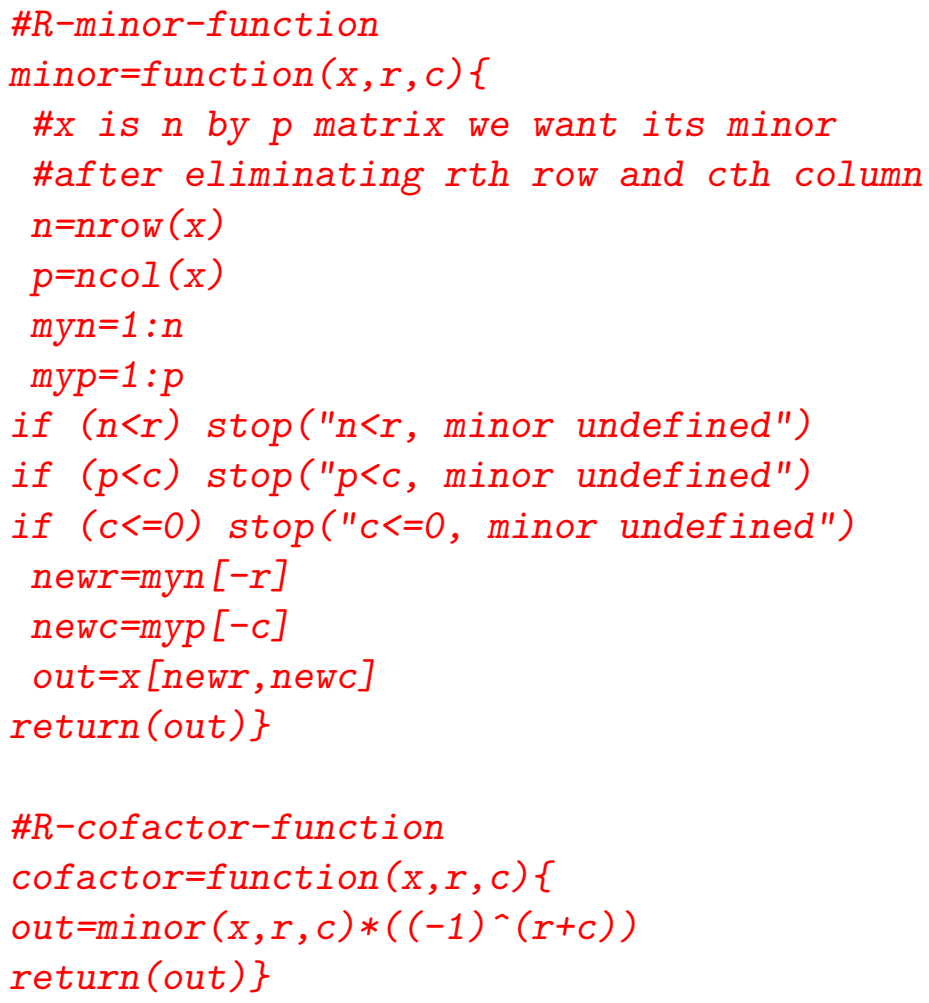


Recall that eq. (16) of my CSSC paper defines the partial correlation coefficients for the $R^{*}$ matrix. The function 'parcor.ijk' computes those partial correlation coefficients bet $X_{i}$ and $X_{j}$ after removing the effect of all $X_{k}$ variables. The function reports a list of removed columns named 'myk'.

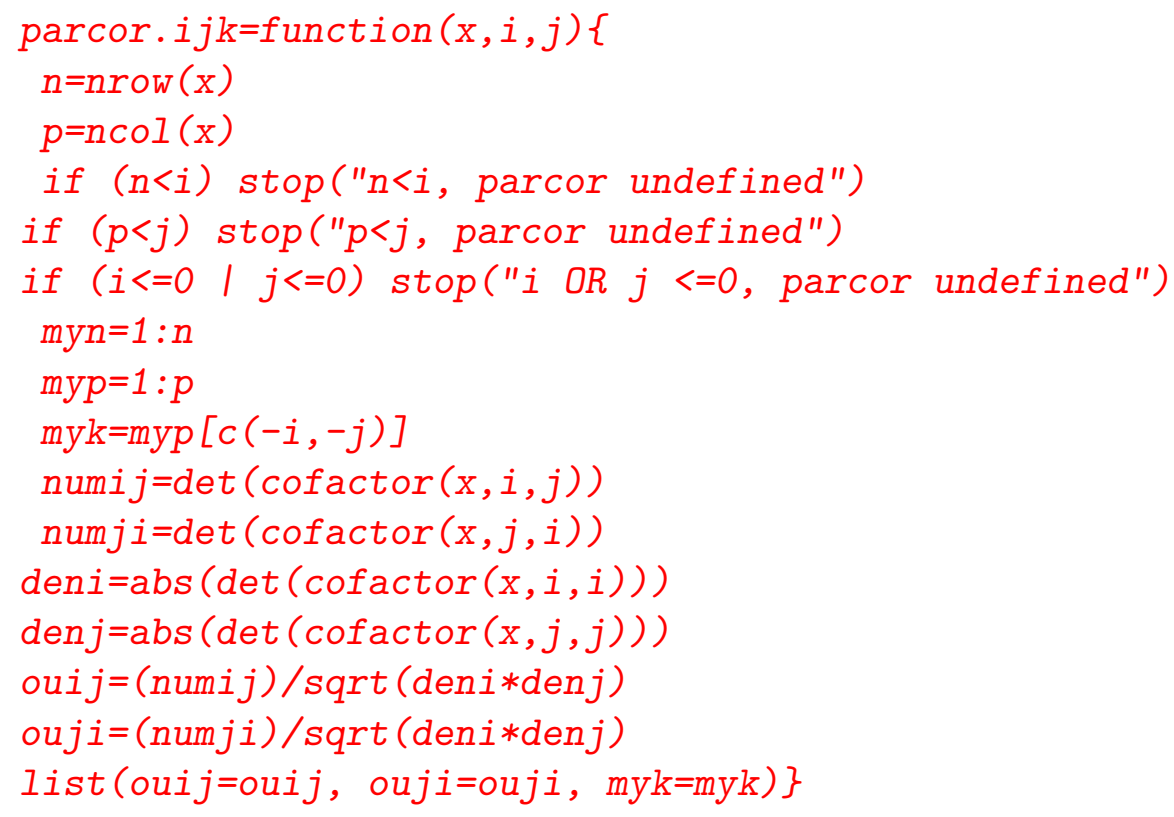

Instead of using the general function 'parcor.ijk', it is convenient to use Cox-Vermuth function called 'partialc2' to get the betas or scale free partial regression coefficients described in my CSSC paper using the EcDev data.

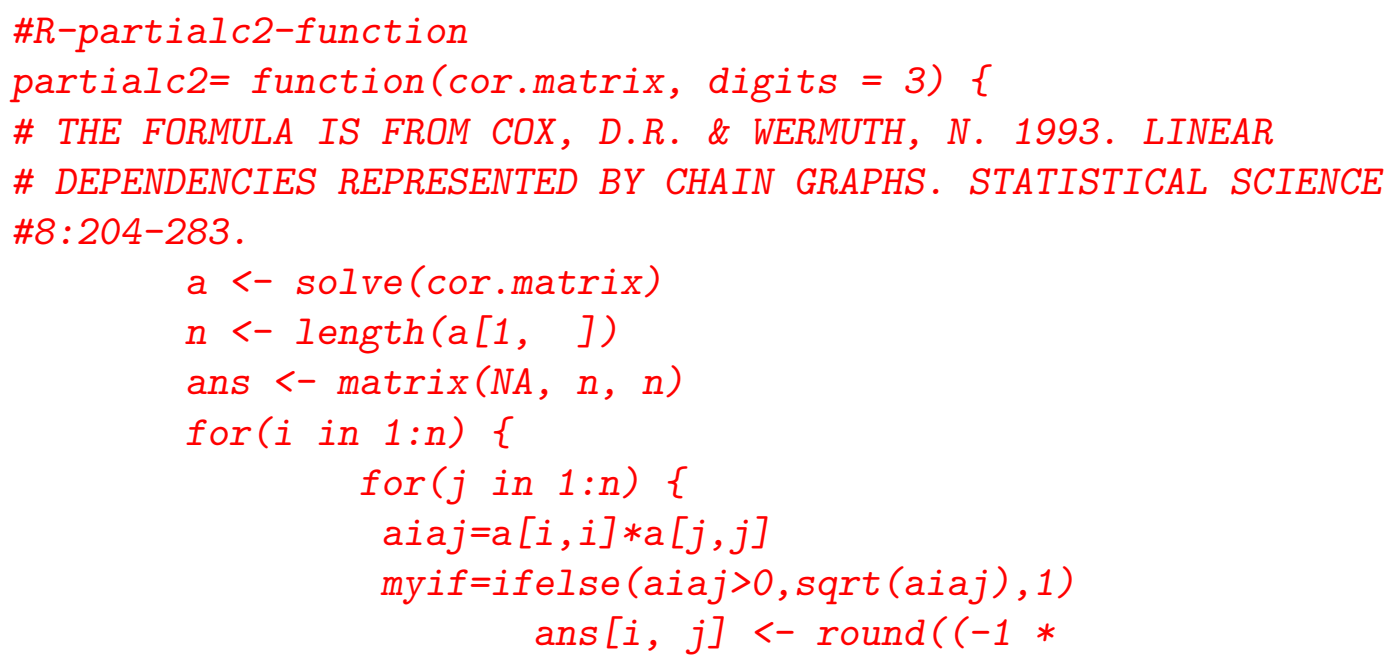


$\operatorname{return}(a n s)\}$

$$
\begin{aligned}
& a[i, j]) / m y i f, \\
& \operatorname{digits)\} \} }
\end{aligned}
$$

The function 'getbeta' computes the betas or scale-free regression coefficients of two variables after removing the effect of additional variables. If the option 'rstar' is set FALSE, then Pearson correlations are used, which in tern require the function 'rcorr' of the package 'Hmisc'.

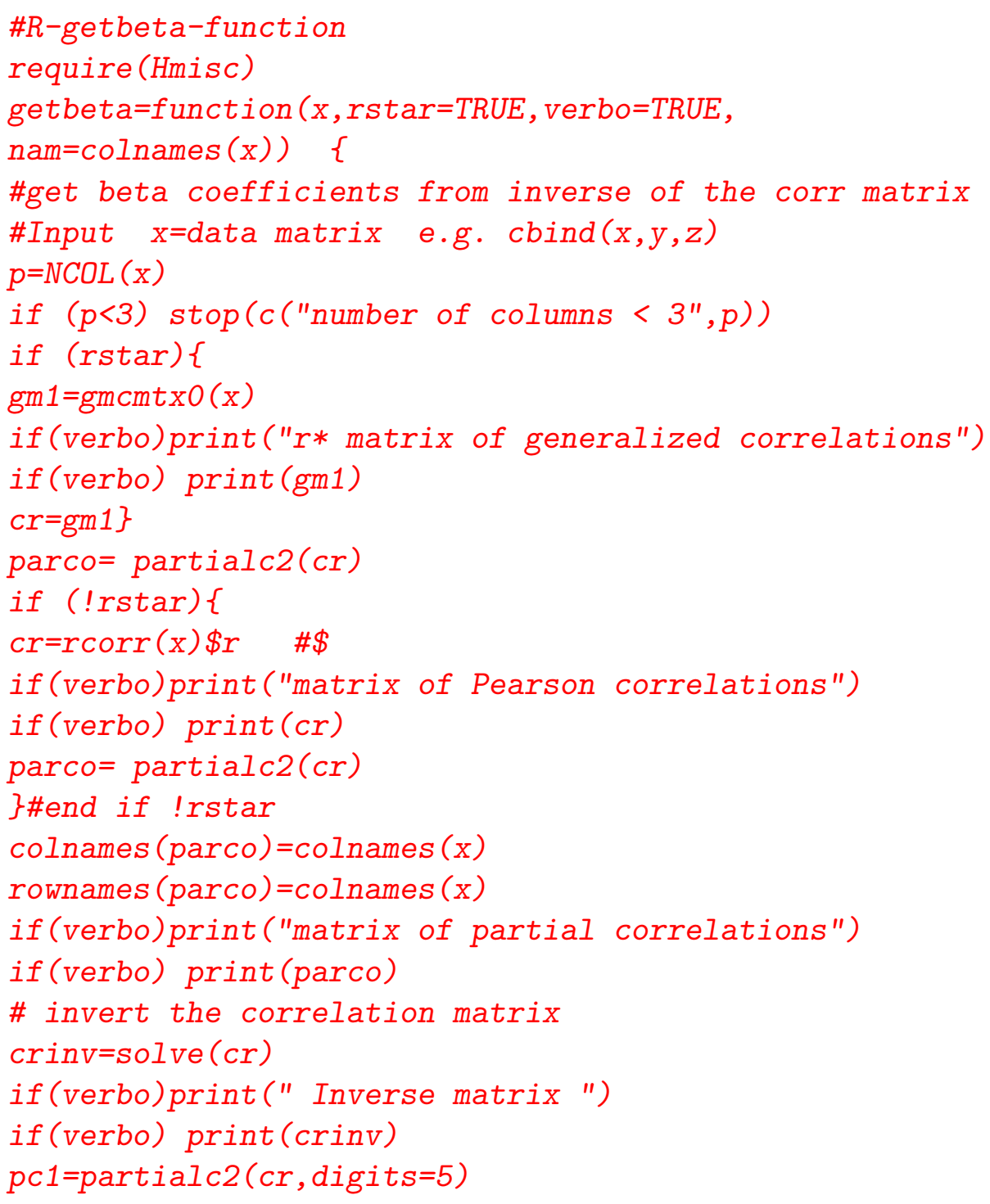




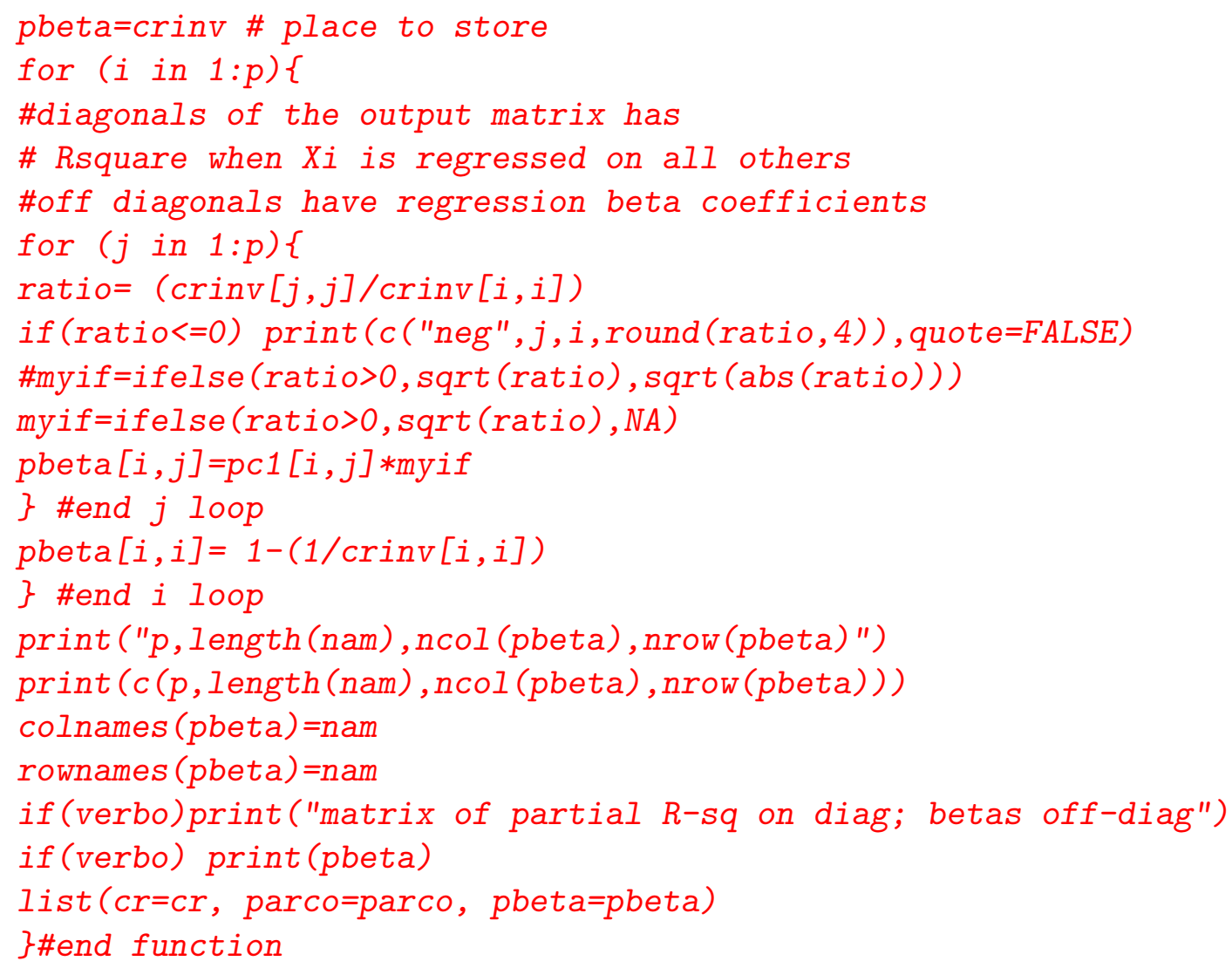

Now we turn to recreating Table 7 of the CSSC paper. It is conveniently created by using the additional functions 'partialc2' given above and 'getParcor' given below.

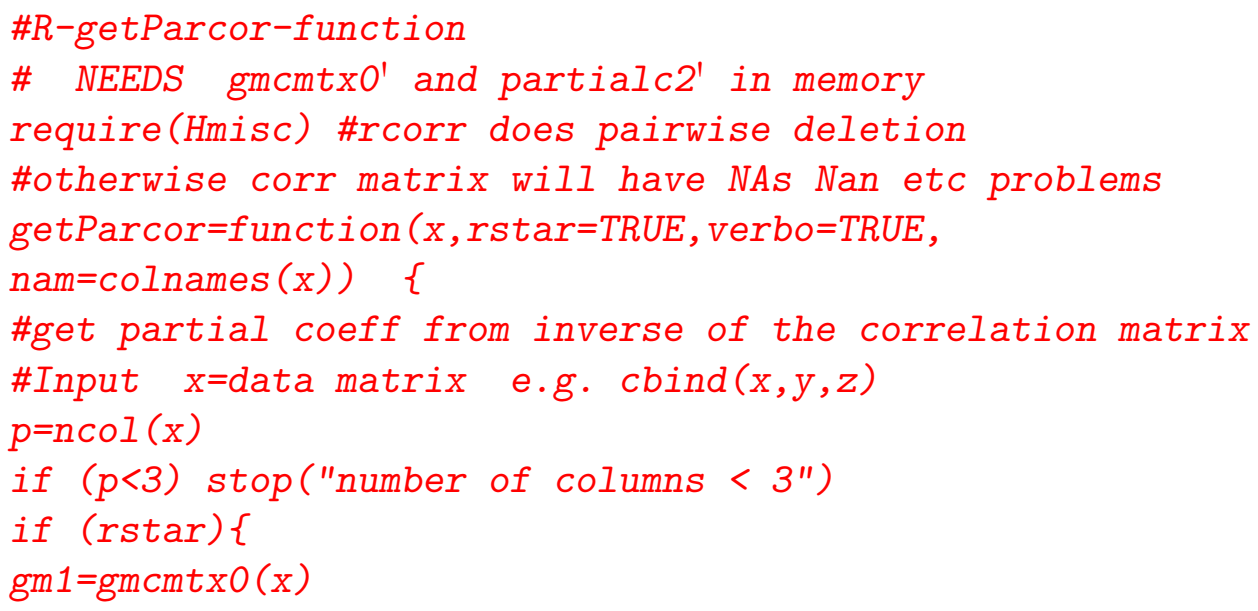




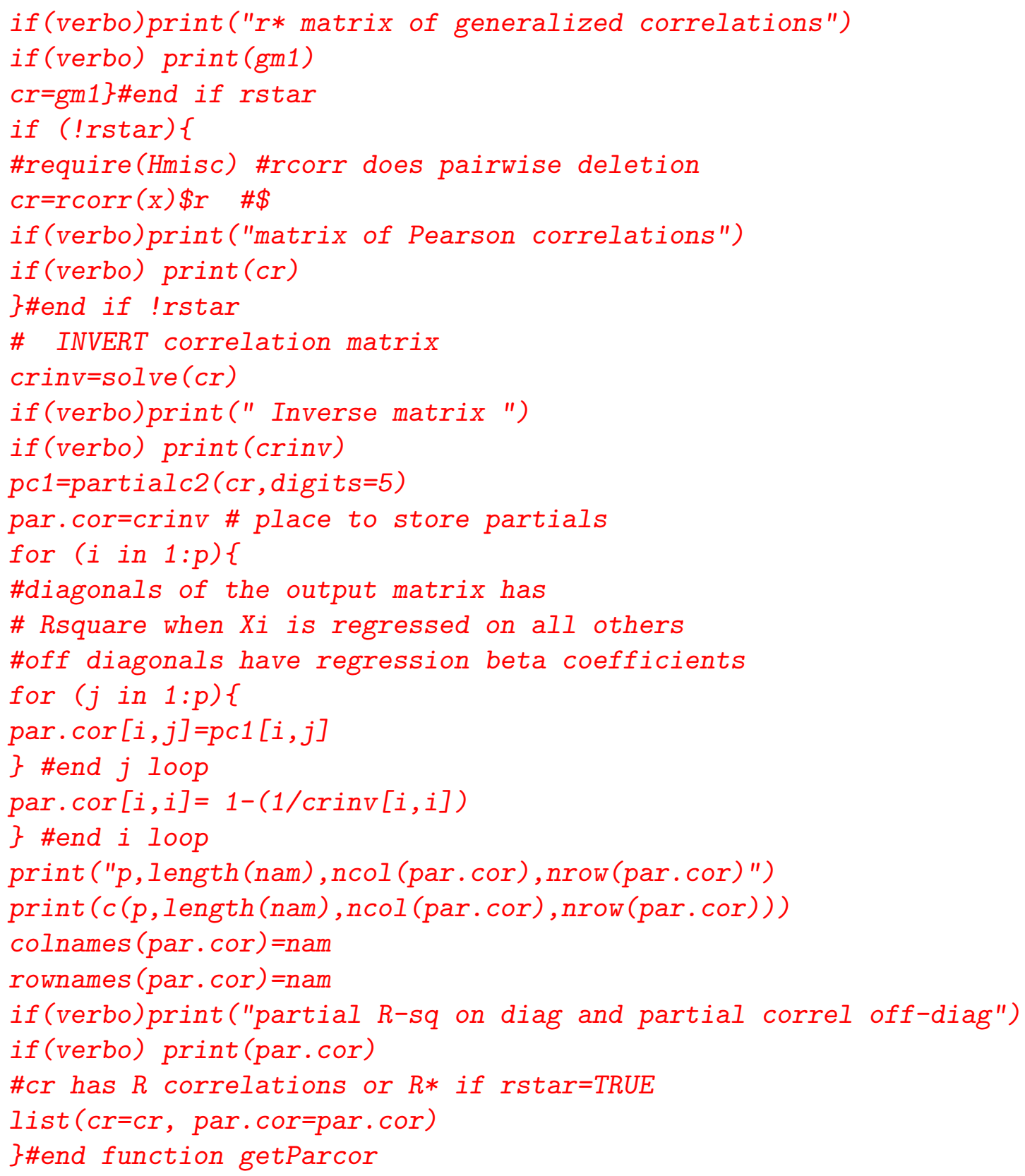

Now we are ready to apply the above tools to Economic Development data to create Table 7 of my CSSC paper illustrating the use of partial correlations and betas in multivariate situations. Table 7 has two vertical panels with the left panel for the three variables my3=(MPI, GRO, GINI) 
and the right panel is for four variables my $4=(\mathrm{MPI}, \mathrm{GRO}, \mathrm{GINI}, \mathrm{GEI})$. Recall that the 'getbeta' function offers a choice of Pearson correlation by using the command ' $\mathrm{g} 1=$ getbeta(my3,rstar=FALSE)' illustrated below. Of course, we are more interested in the new $R^{*}$ and betas obtained from it obtained by the command: 'g2=getbeta(my3,rstar=TRUE)'. The four rows of the left panel on Table 7 of the CSSC paper are in the matrix 'myx'.

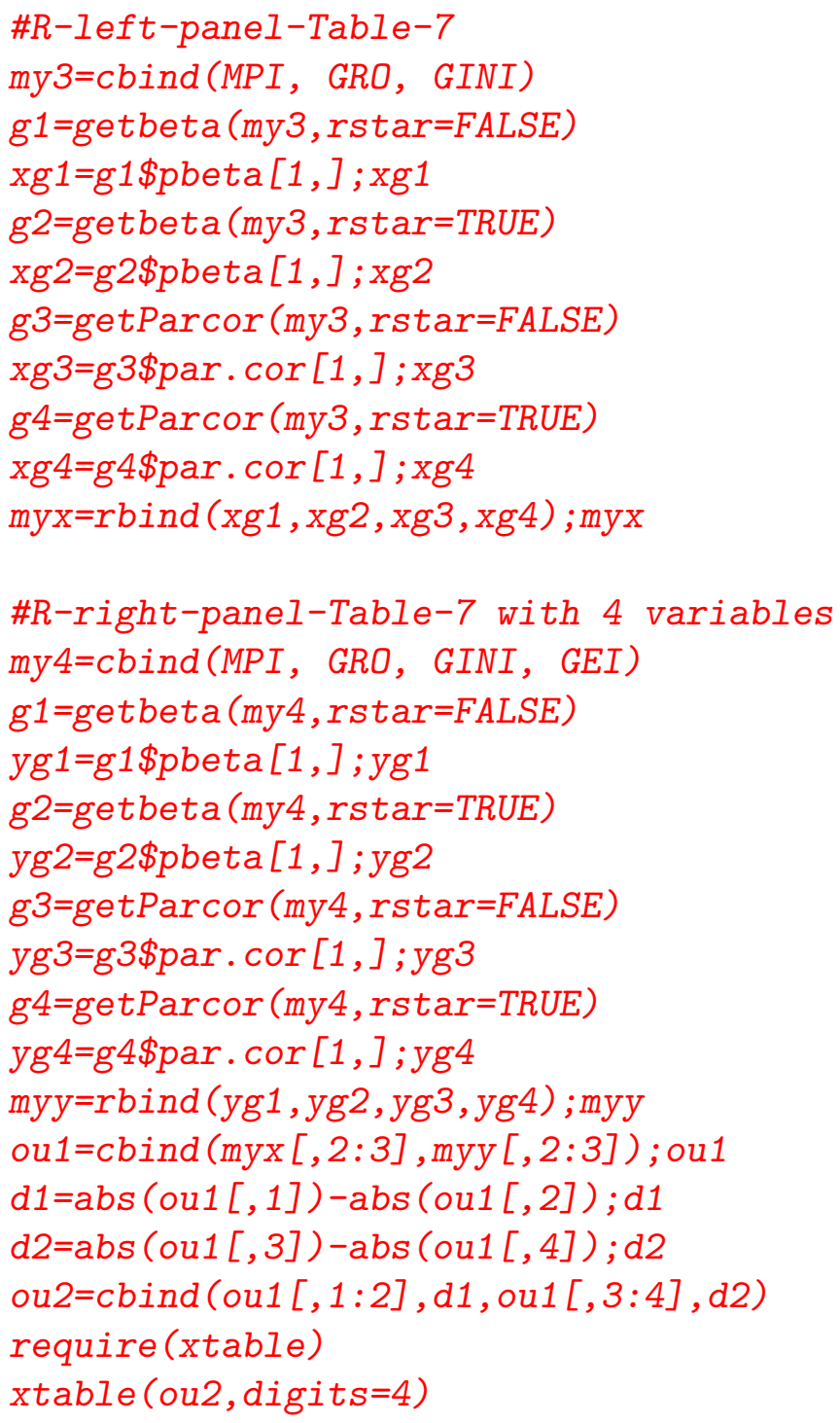

The output from the above code creates Table 7 in my CSSC paper, where the discussion of evidence supports Bhagwati's growth policies over Sen's 
redistribution policies for economic development. It allows consideration of equation (22) (regressing MPI on GRO and GINI) which has two regressors illustrating multivariate regressions. By using partial correlations we can focus on the effect of one regressor at a time, removing the effect of remaining regressors. The right panel of Table 7 considers regression of MPI on GRO, GINI and GEI (i.e., three regressors). The partial correlations in the right panel remove the effect of two regressors.

\section{Non-spherical Error Corrections}

Correcting for autocorrelation and heteroscedasticity (non-spherical errors) is readilty done by using the following function called 'autohetero', which in turn needs a function 'sort.matrix' which is given first. The theory is described in Vinod (2010). The basic idea is to use generalized least squares.

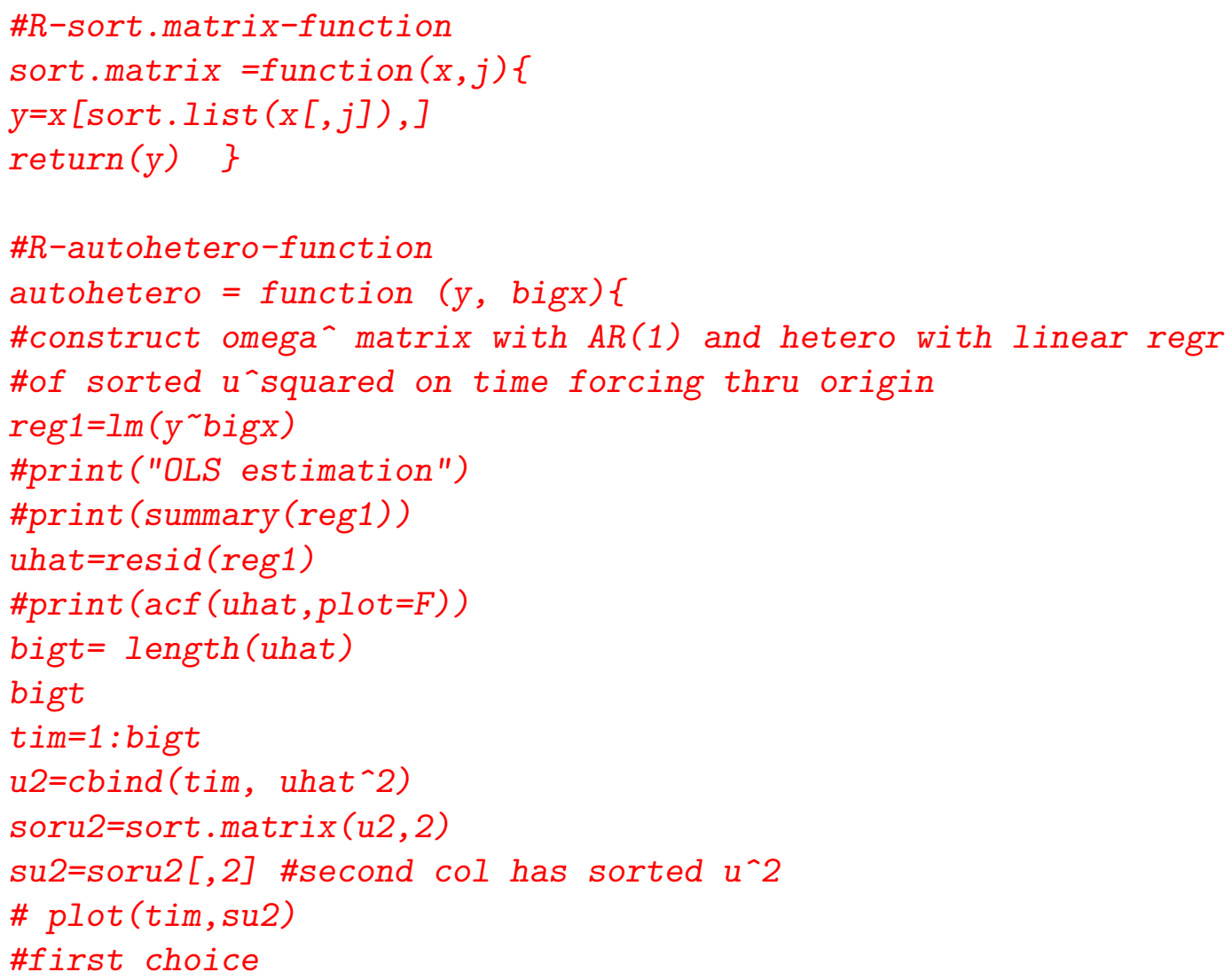




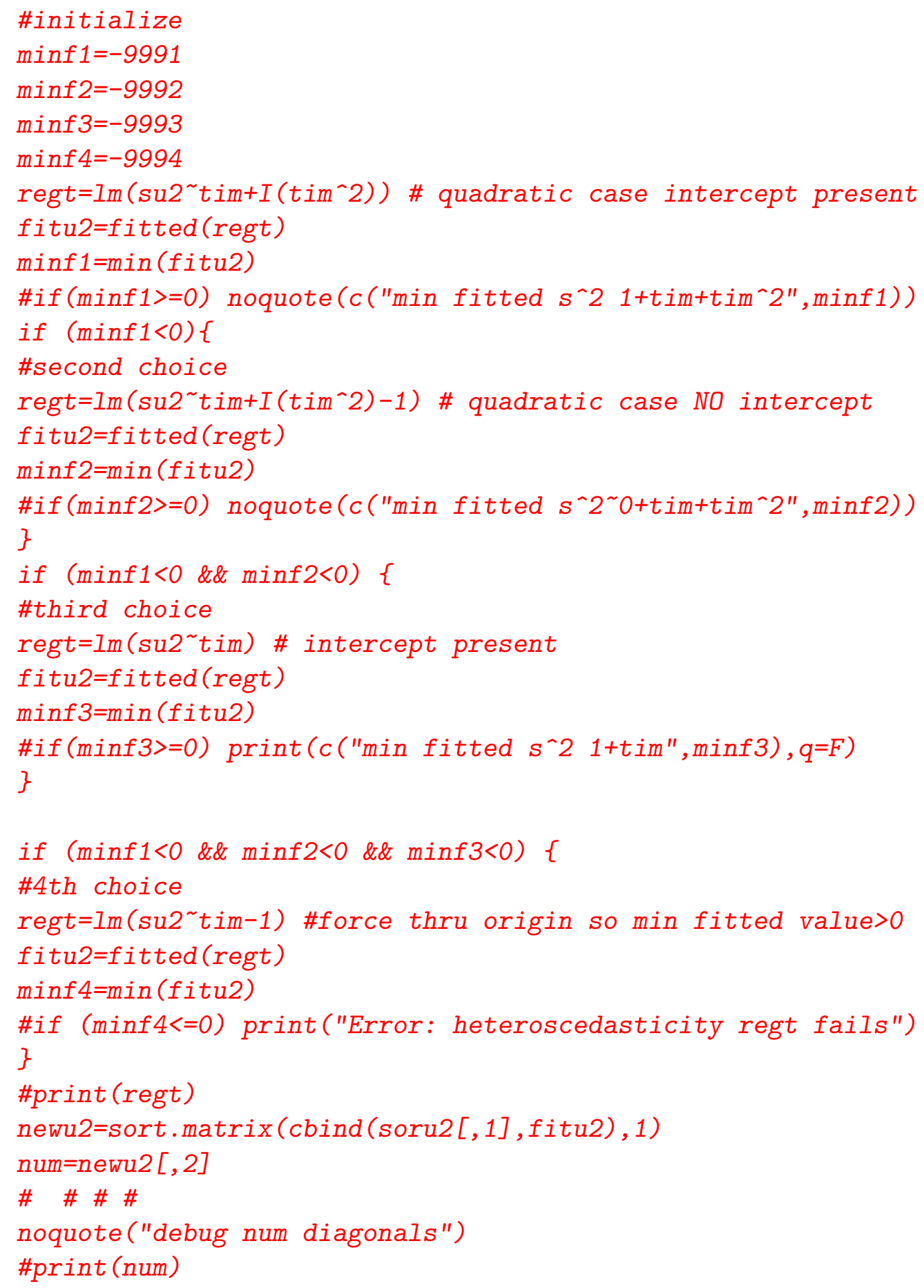




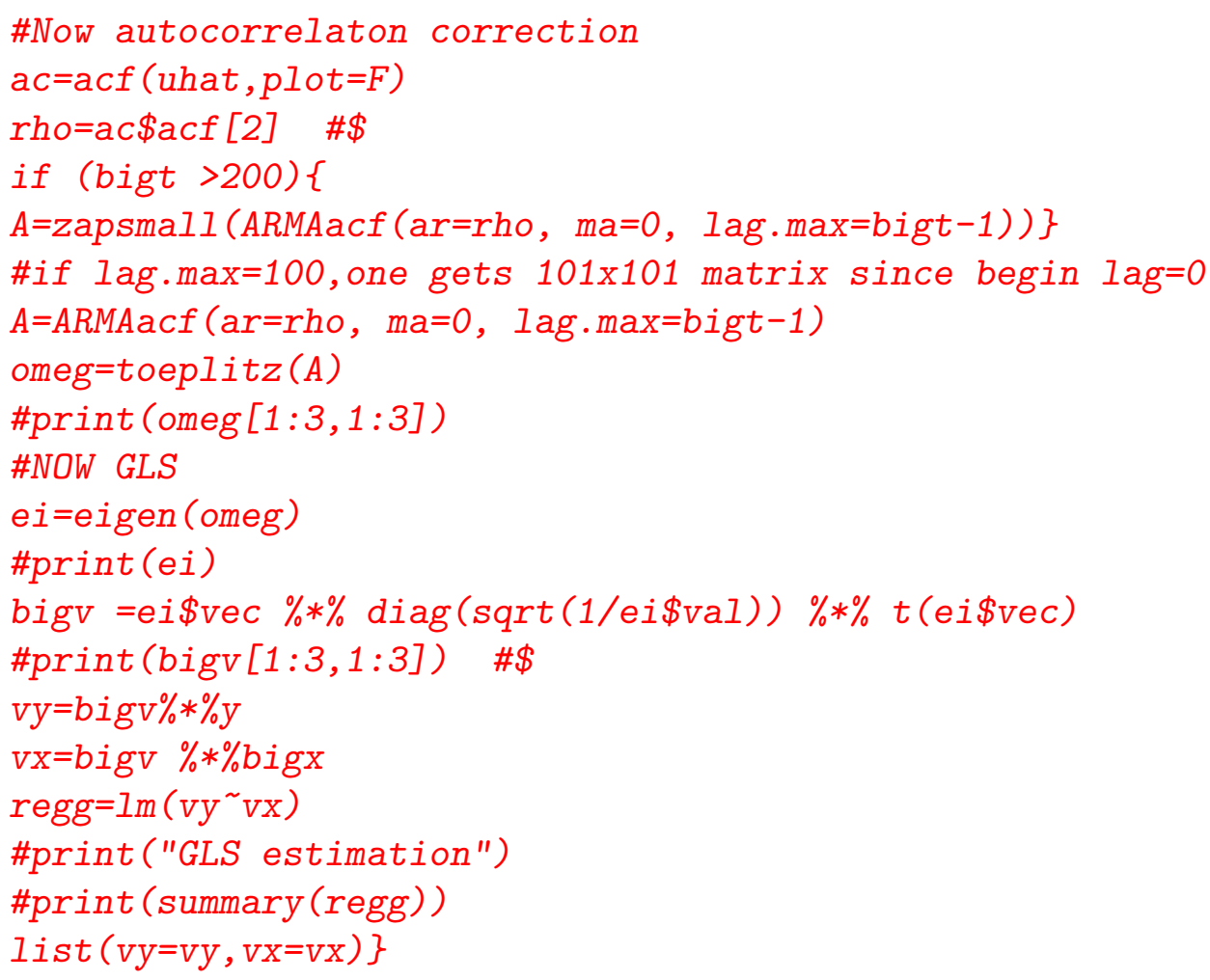

\section{Sampling distribution of $\hat{\delta}$ using meboot}

The maximum entropy bootstrap is implemented in the $\mathrm{R}$ package 'meboot'. It creates a large number $(\mathrm{n} 999=999)$ of resamples of ' $\mathrm{x}$ ' (similar data series) by the command ' $x$ boot $=\operatorname{meboot}(\mathrm{x}=\mathrm{x}$, reps $=\mathrm{n} 999) \$$ ensemble'. Now xboot has 999 columns of data. The following code needs 'gmcxy.np' from Section 6.

The function 'pcause' computes $\mathrm{P}$ (cause) defined in eq. (9) of my CSSC paper giving the larger of the two rejection probabilities. The computed $\mathrm{P}$ (cause) lies in the range $[0,1]$, where a larger value is more desirable for inference about the causal direction based on $\hat{\delta}$ using the meboot bootstrap.

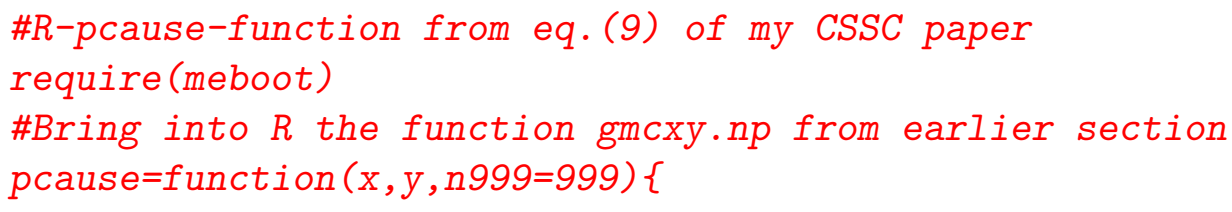




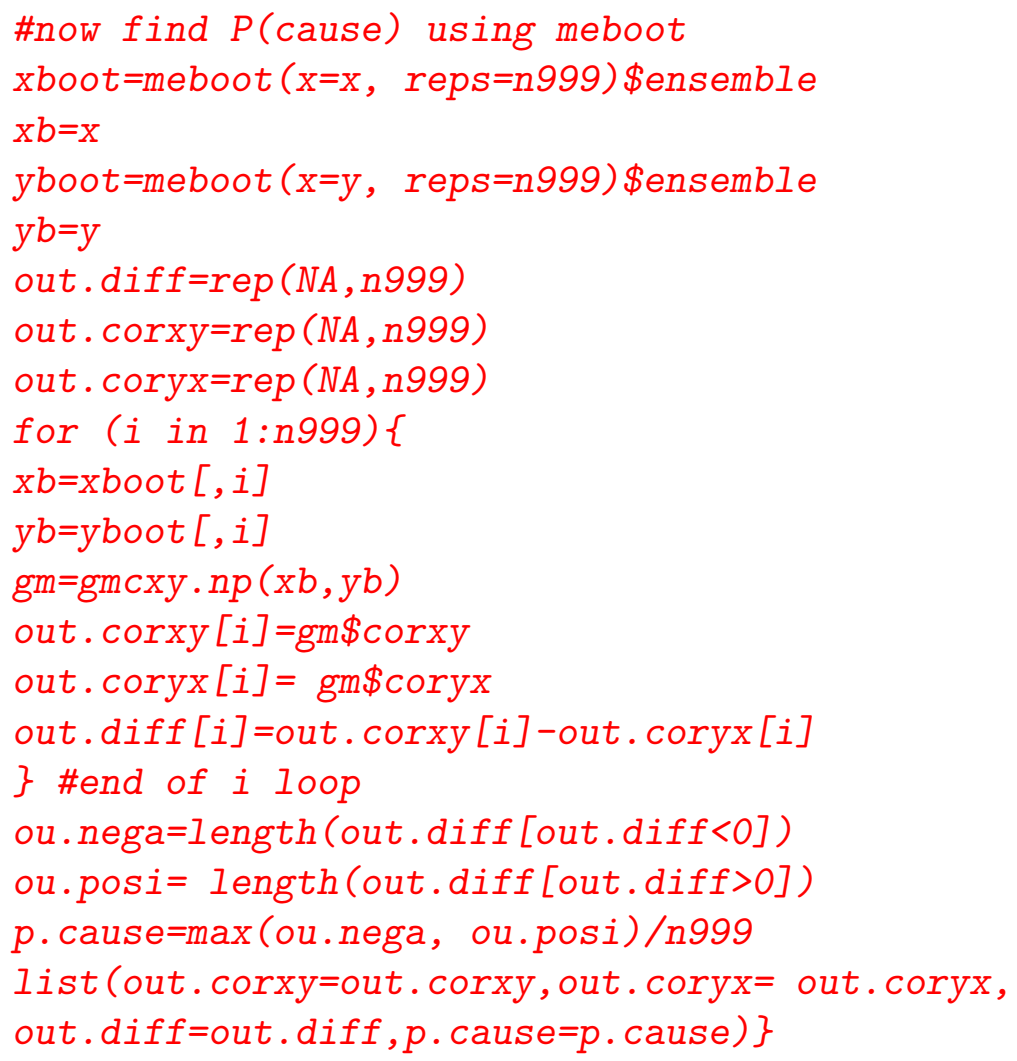

Note that 'out.diff' contains 999 estimates of $\hat{\delta}$ allowing us to approximate its sampling distribution.

\section{Comprehensive Tables Displaying 36 Rows for 9 Variables}

Note that our kernel causality criterion relies on the asymmetry of the generalized correlation matrix $r^{*}(i, j)$ to identify the causal variable. Since the row variable $X_{i}$ is the "effect" and the column variable $X_{j}$ is the "cause," we identify the cause depending on which matrix entry $(\mathrm{i}, \mathrm{j})$ or $(\mathrm{j}, \mathrm{i})$ is larger. EcDev data have a large $9 \times 9$ matrix and it is not convenient to manually compare such entries.

It is safer to let the computer choose all unique $(i, j)$ pairs and explicitly identify the cause by comparing the indicated entries of the asymmetric matrix. Since $\left({ }^{9} C_{2}=36\right)$, our EcDev data needs a comparison of 36 pairs. 
Tables 4 to 6 of my CSSC paper do have 36 rows for each unique pair and the cause is identified and named in the third column by using a computer program. A typical program is given below.

If the bootstrap is used for inference, a much slower version of gmcmtx0 called gmcmtx needs to be used before we do the 36 pairs. Hence we begin with that code.

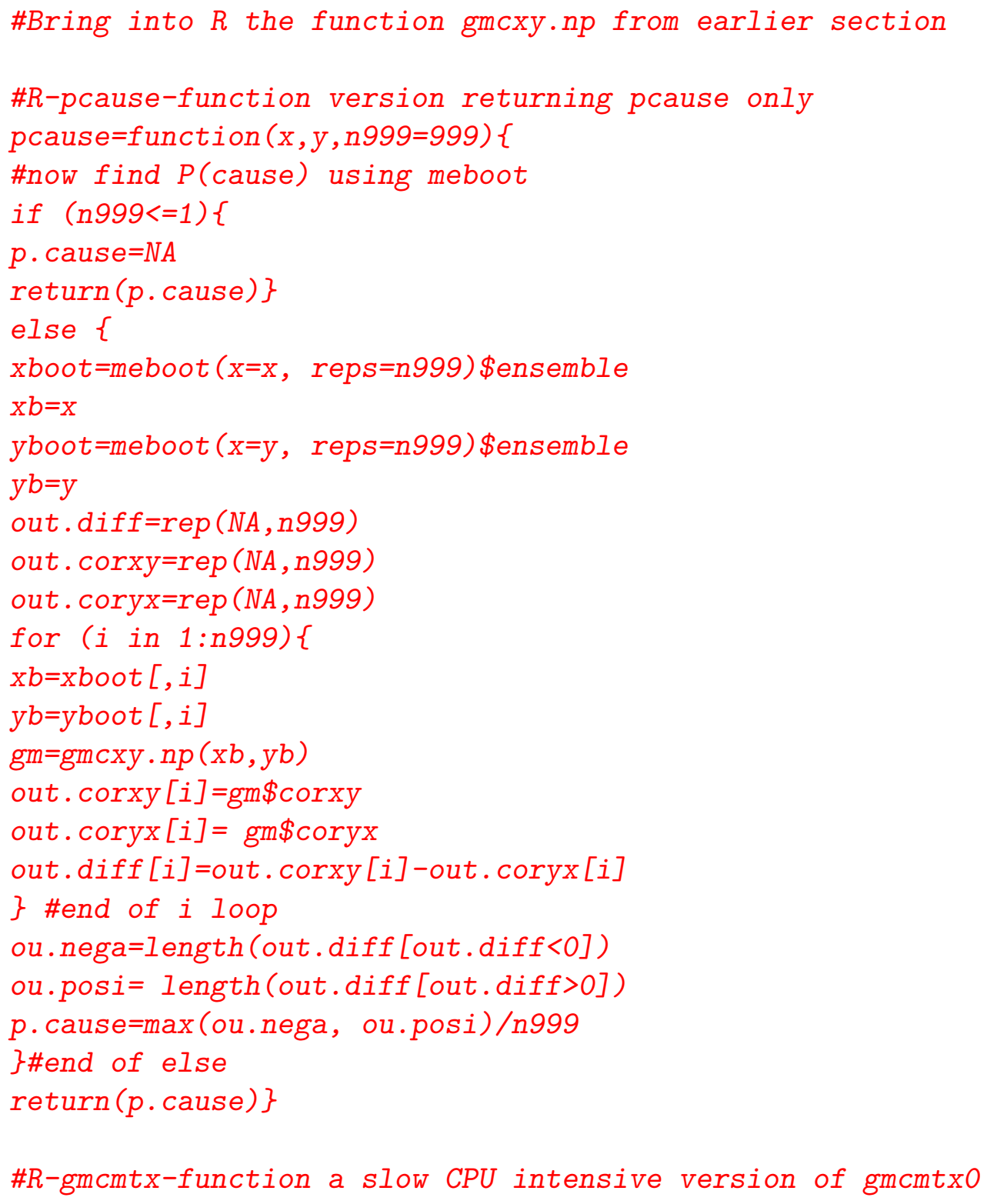




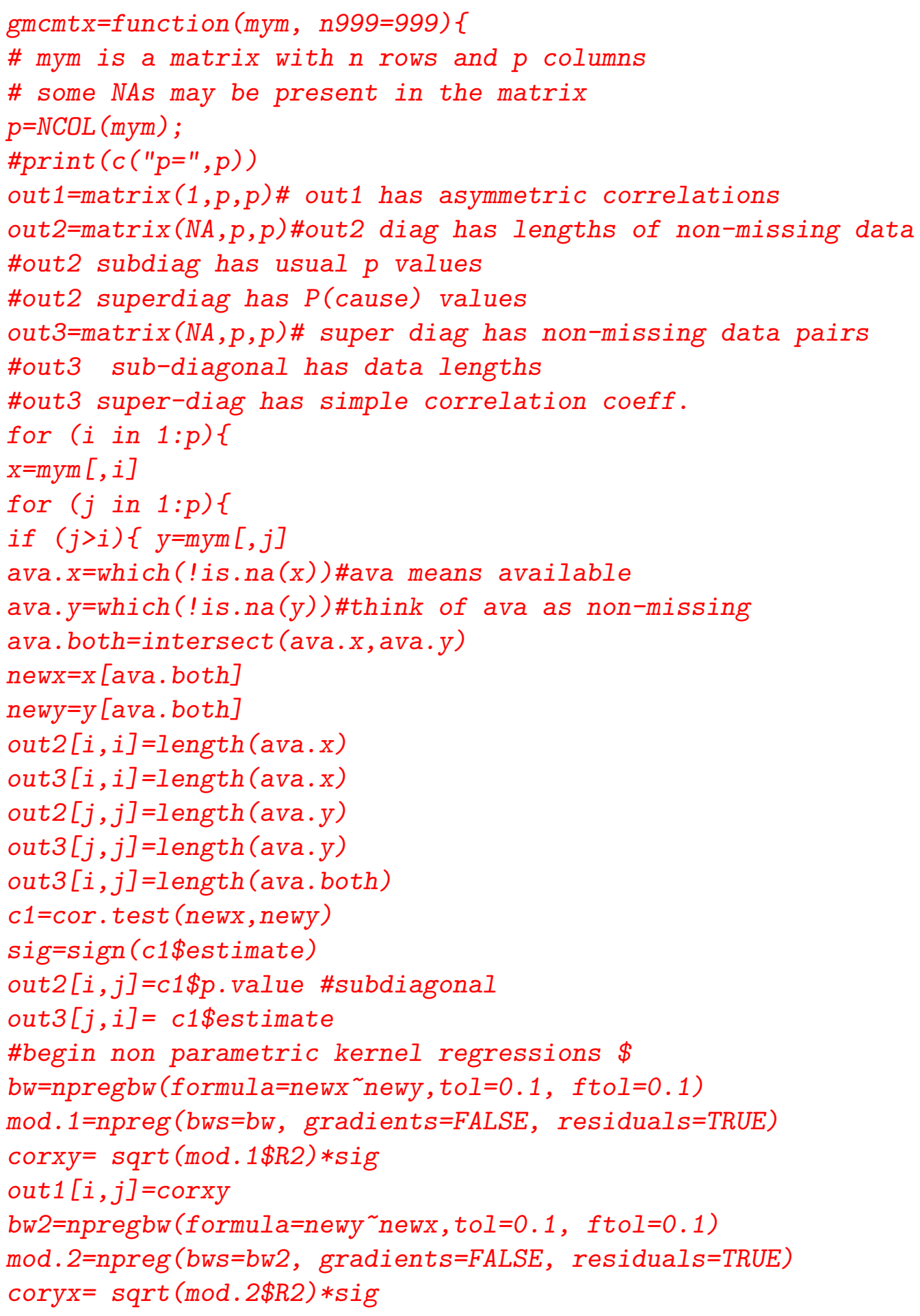




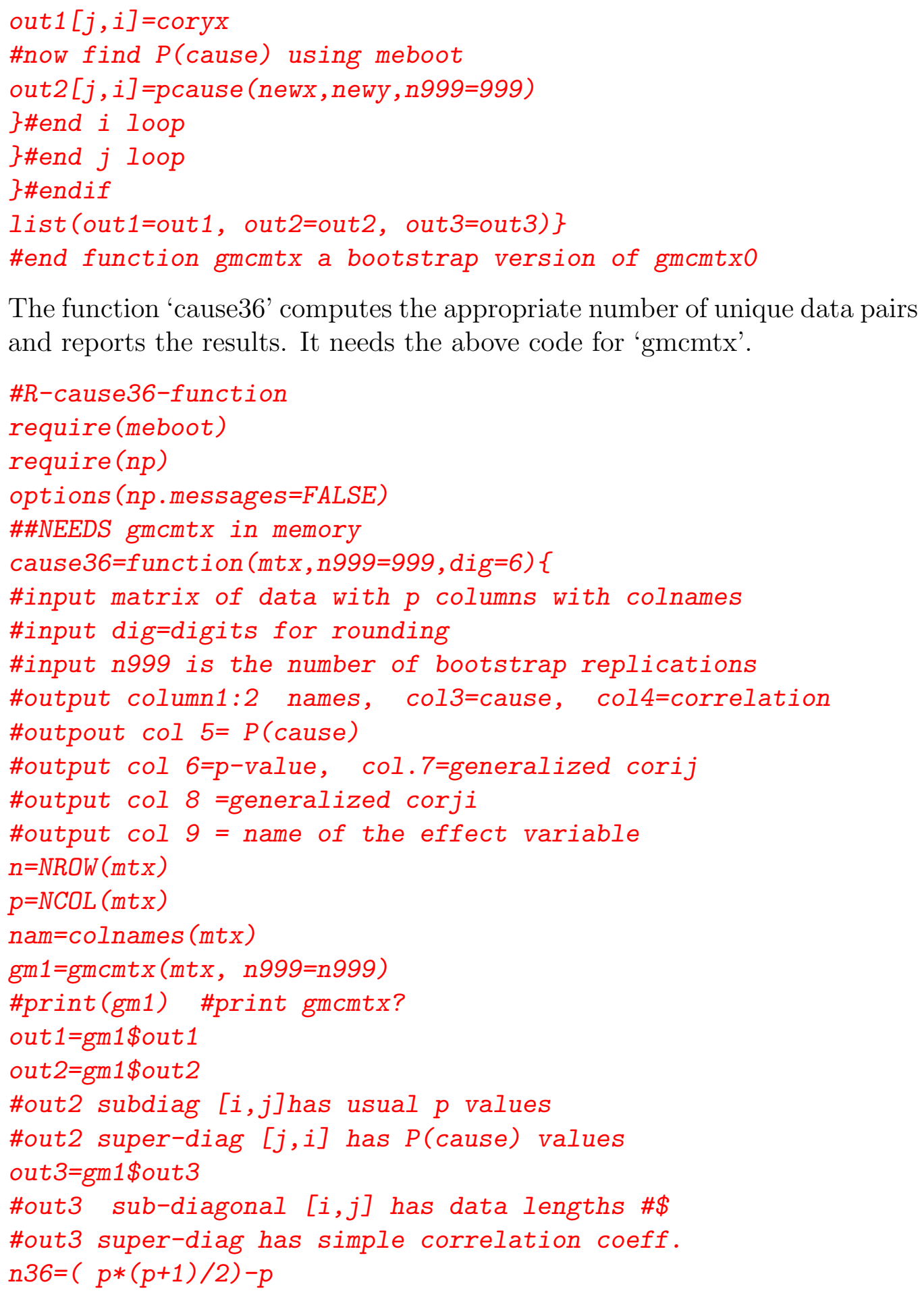

The function 'cause36' computes the appropriate number of unique data pairs and reports the results. It needs the above code for 'gmcmtx'. 


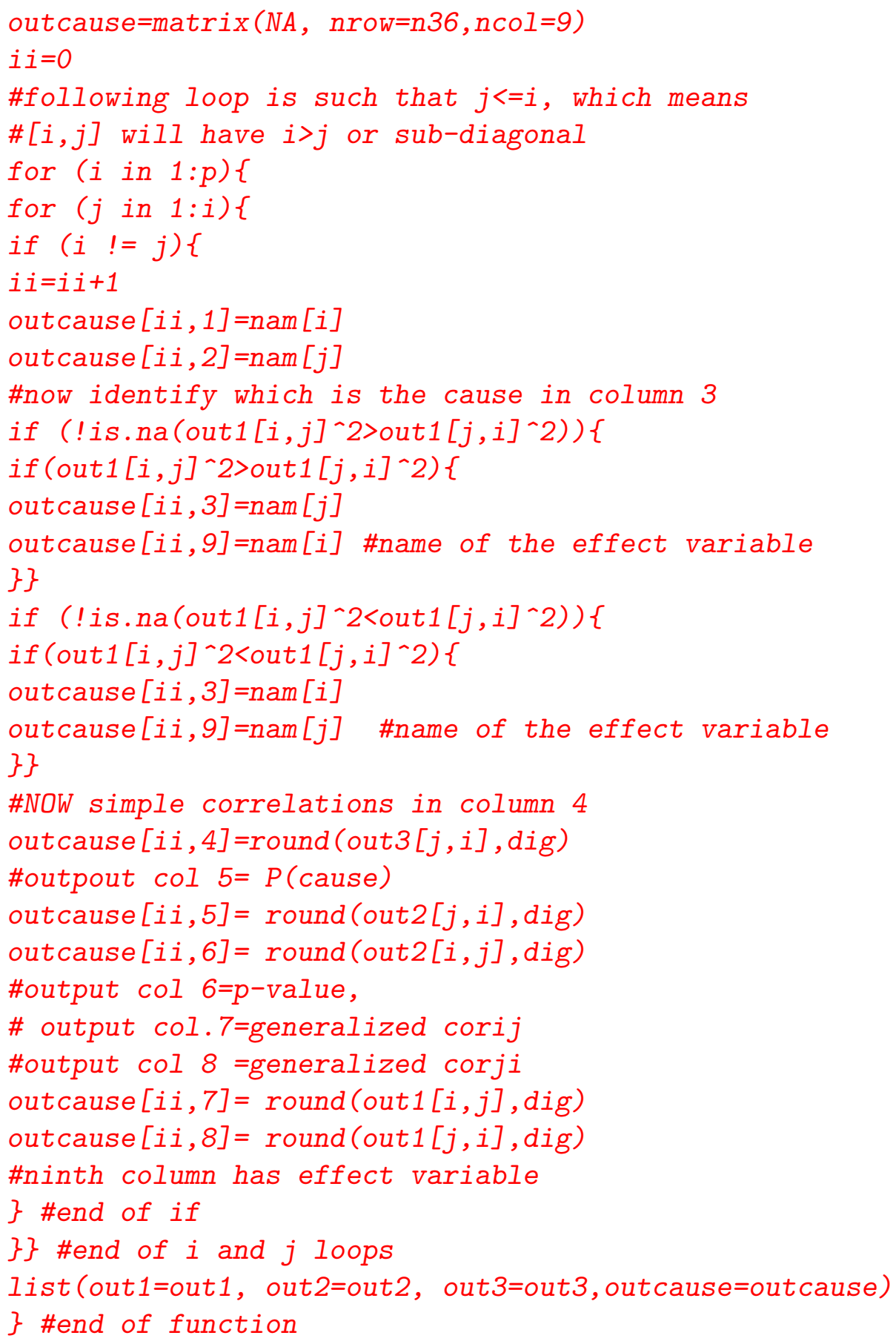

The above code can be modified for various comparisons in Tables 5 and 6 
of the CSSC paper as shown in Section 12 .

\section{Bar Chart of the Frequency of Cause Designations for Nine Variables}

The 'table' command of $\mathrm{R}$ computes the frequencies and its result can be used for a bar plot using the following code named 'R-barchart-EcDev.'

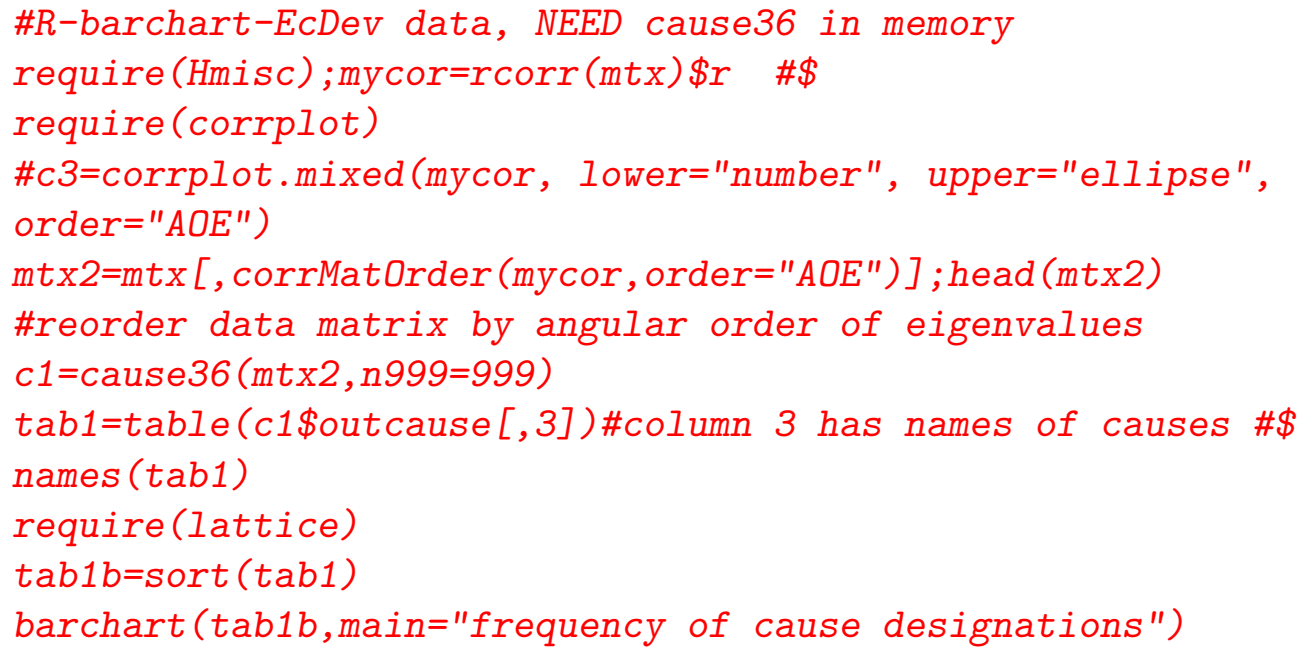

\section{Code for Kernel Regression Robustness Checks}

Recall that if the model regressing $Y$ on $X$ is more robust than the model regression $X$ on $Y$ we conclude that $X$ kernel causes $Y$. Section 2.3 of my CSSC paper describes Kernel Regression Robustness Checks where the first robustness check uses a nonparametric test of dependence between residuals and regressors, where low dependence is desirable.

Table 5 of CSSC paper illustrates the results of this robustness check for EcDev data. The version of gmcmtx0 in the code named 'R-gmcmtx0npdepfunction' is called 'gmcmtx0npdep.' The 0 in the name refers to fast version (without the meboot) and 'npdep' is the name of the function from the package 'np' used here to assess the dependence of regression residuals with 
the regressor. The dependence is measured by $S_{\rho}$ explained in Section 2.3 of my CSSC paper. Note that it is desirable to choose the model with the "smaller" value of $S_{\rho}$.

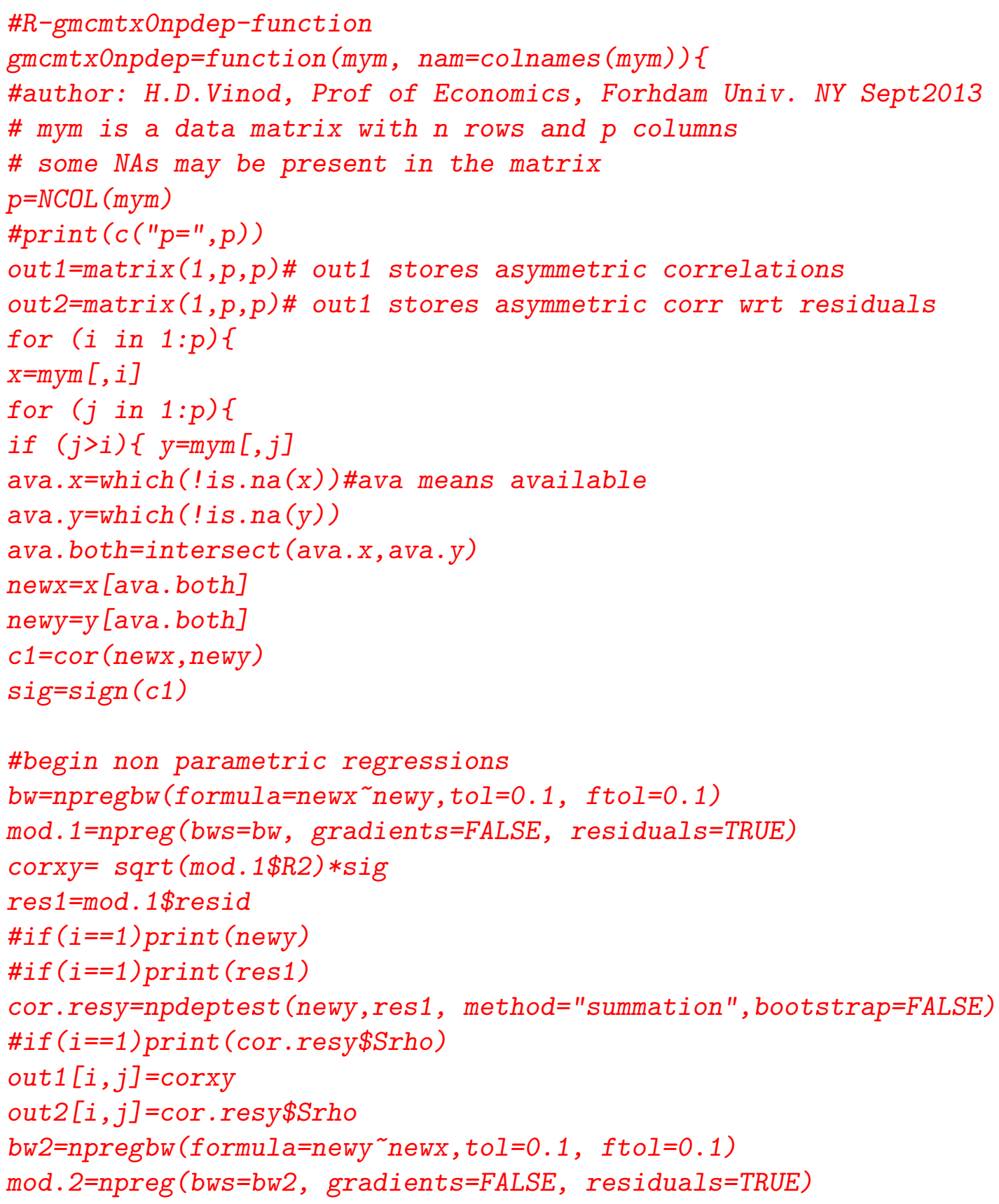




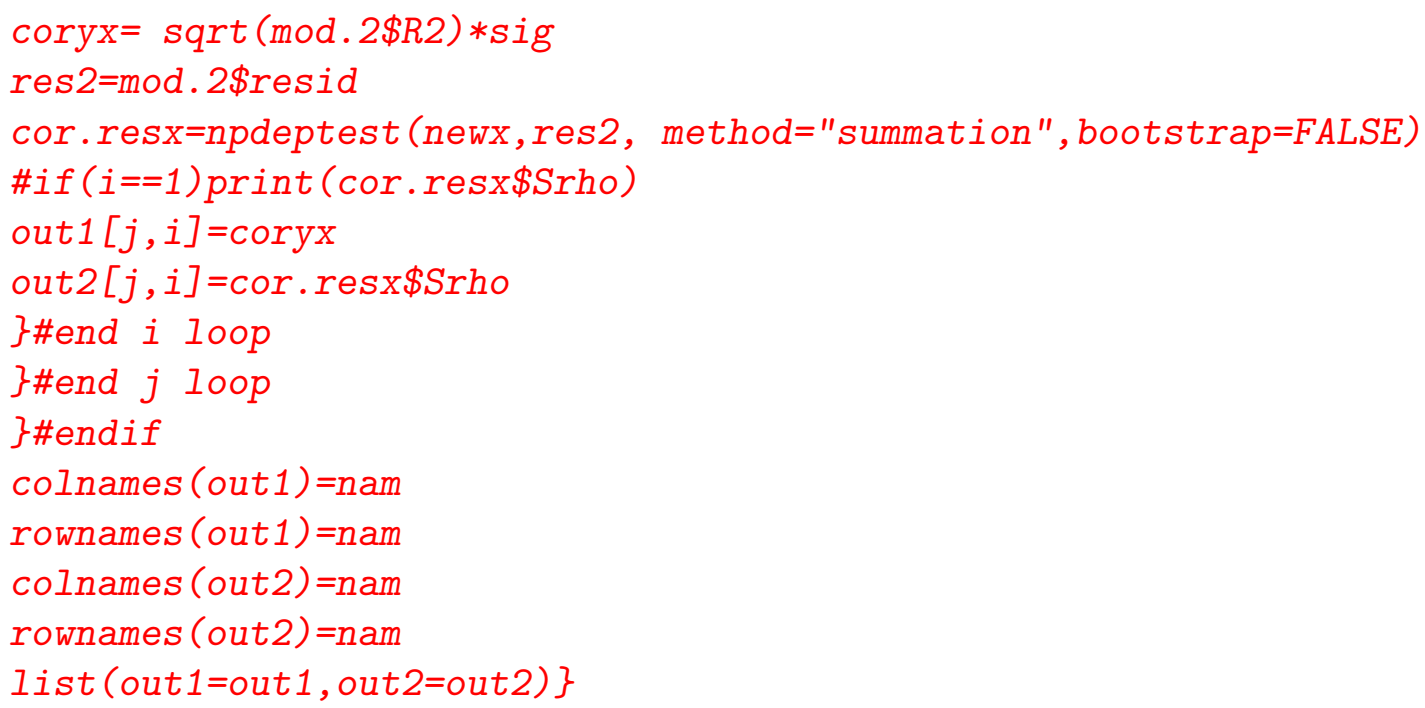

The command 'gm1=gmcmtx $(\operatorname{mtx}, \mathrm{n} 999=\mathrm{n} 999)$ ' in the above code for the function 'cause36' should be replaced by 'gm1=gmcmtx0npdep(mtx)' to create a Table similar to Table 5 in my CSSC paper.

Section 2.3 of the CSSC paper also describes another robustness check based on out-of-sample forecasts. The $\mathrm{R}$ code for that purpose is given next.

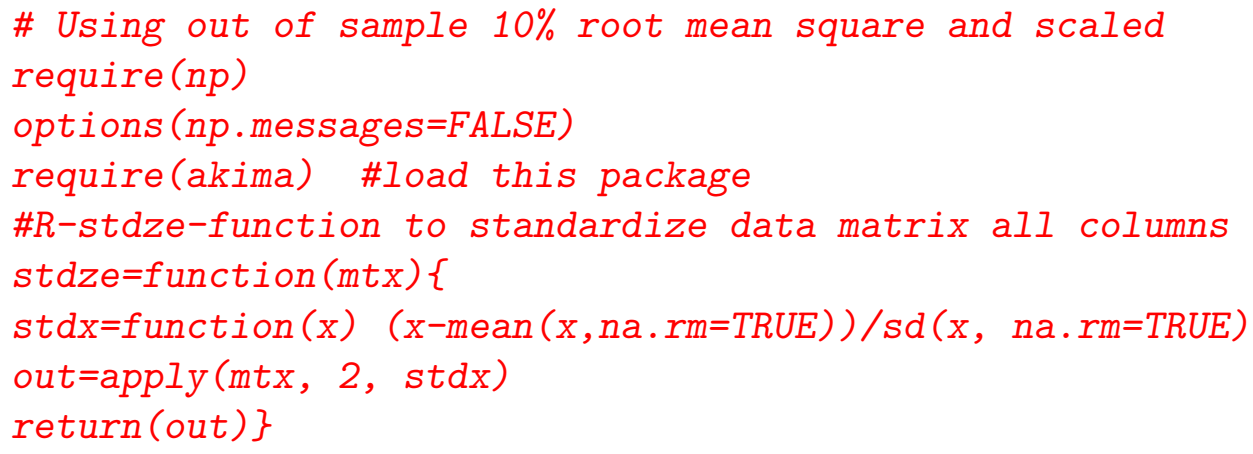

The above standardization makes the mean zero and standard deviation unity for all columns. The following function computes root mean squared (rms) values defined in Section 2.3 of the CSSC paper. It uses the standardization function and then sorts on the first column, while carrying along all other columns.

$\# R$-getrms-function computes root mean squared of errors getrms $=$ function $(x, y, \operatorname{perc}=10$, reps $=10)\{$ 


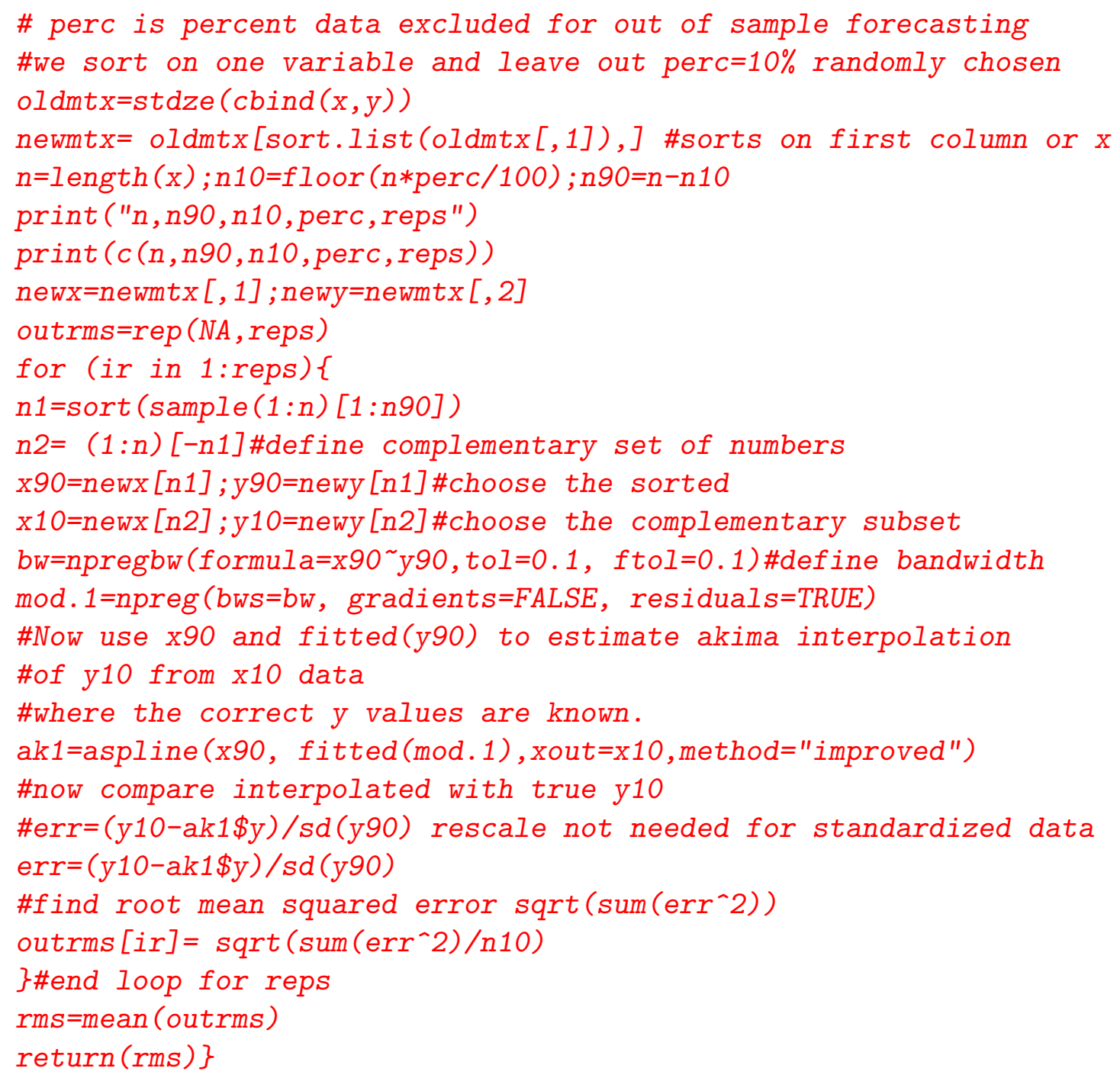

Next, we provide code for implementing robustness in terms of out-of-sample forecasts using a function similar to gmcmtx called gmcmtx0out3samp. The zero in its name refers to fast version without meboot. out3samp in its name refers to a version of out-of-sample forecasting which reports asymmetric root mean squared (rms) values for comparison of the two models.

$\# R$-gmcmtx0out3samp-function $0=$ no boot, \# Using out of sample $10 \%$ root mean square and scaled \#version 3 standardizes data for forecasting out of sample 


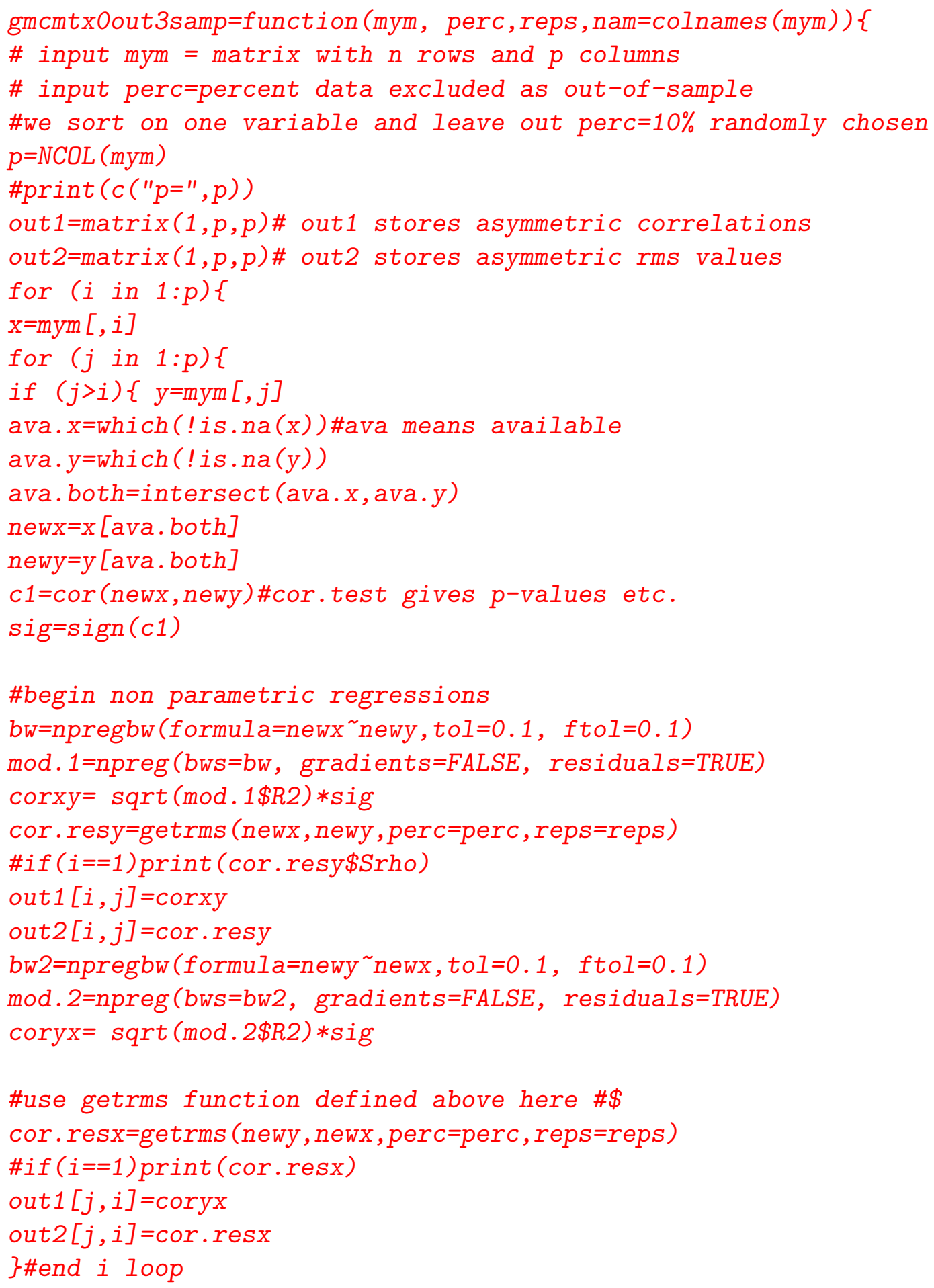




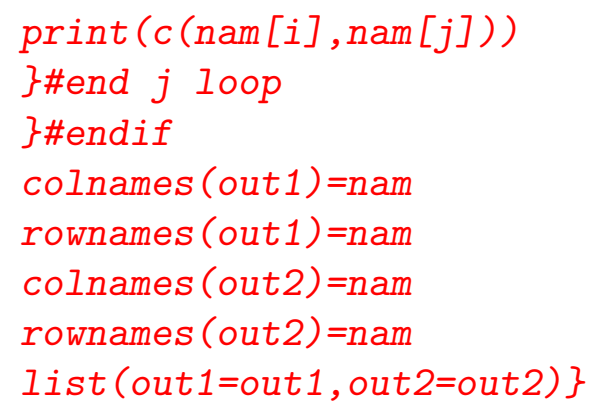

Now we apply the above robustness check to EcDev data having 9 columns and 36 unique pairs. This is done by using a revision of the function cause 36 called 'cause036outsamp', which in turn calls the function 'gmcmtx0out3samp' above.

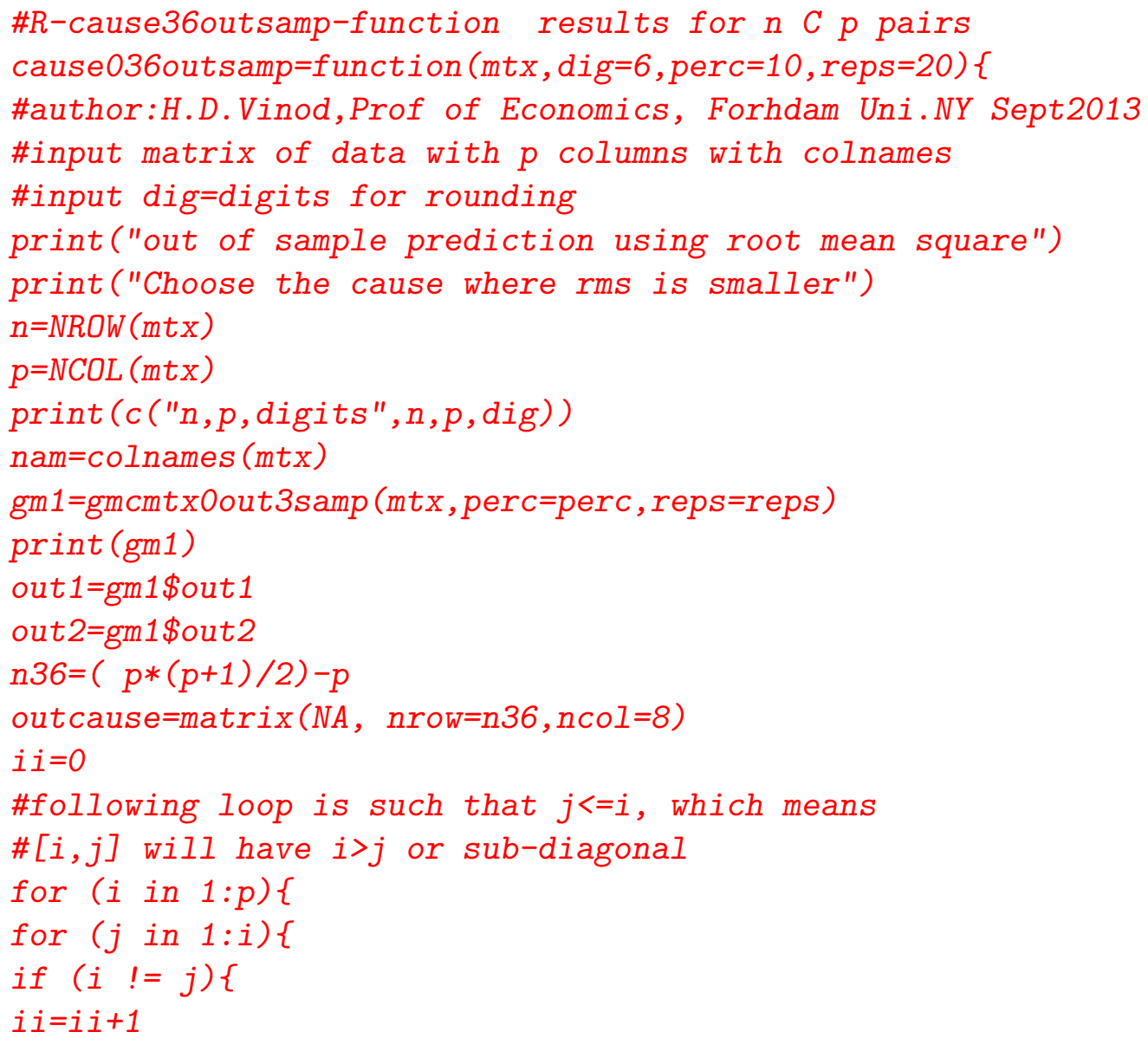




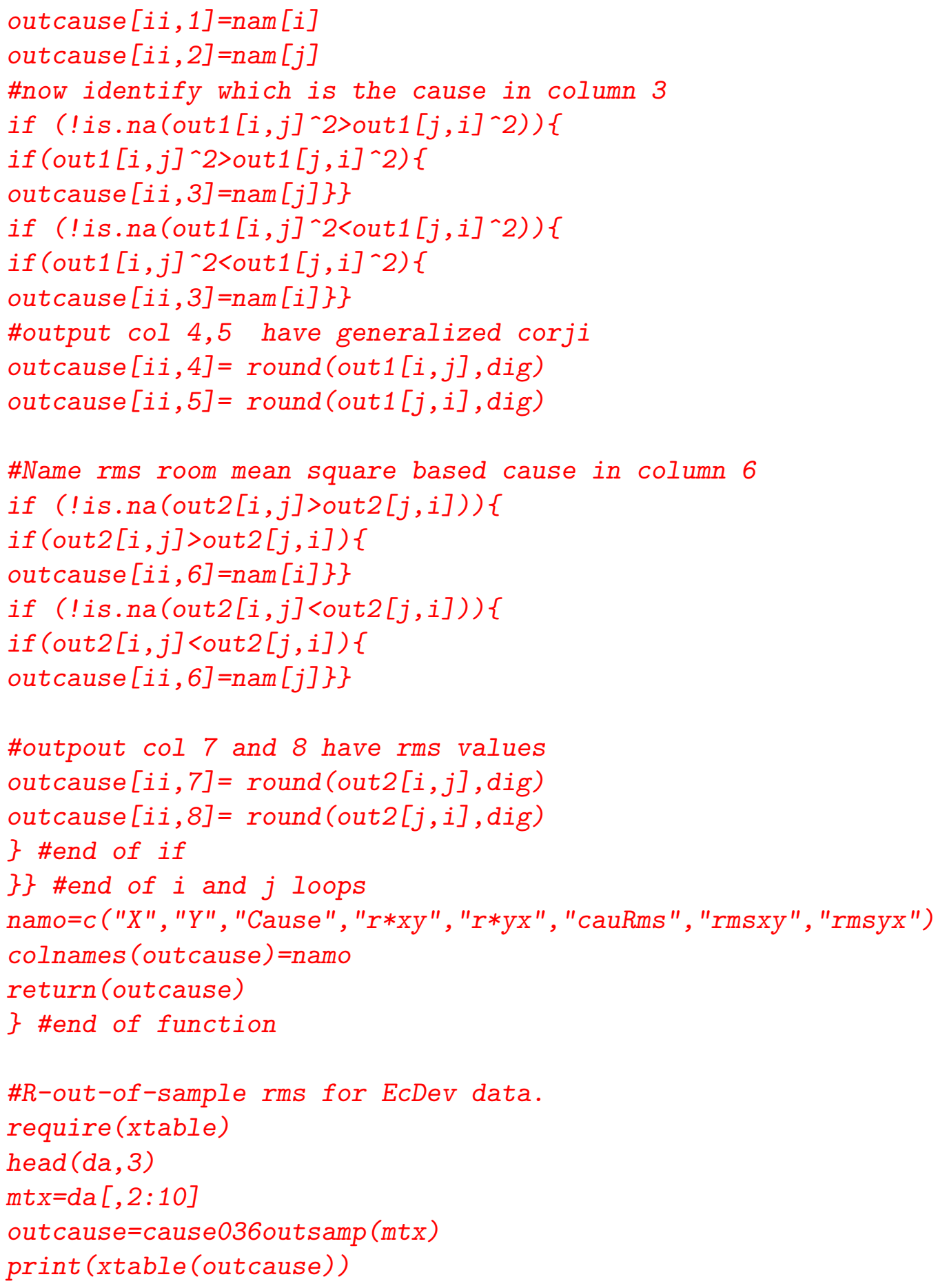




\section{Final Remarks}

The discussion in this supplement is kept at a practical level to facilitate further enhancements, which can be both theoretical and practical. I hope that readers find the supplementary material containing $\mathrm{R}$ software useful. Suggestions to streamline, improve and convert the code into an $\mathrm{R}$ package are welcome, and may be sent by e-mail to vinod@fordham.edu.

\section{References}

Hidalgo, C. and Hausman, R. (2009), "The building blocks of economic complexity," Tech. Rep. 26, Proceedings of the National Academy of Sciences, URL http://www .pnas.org/cgi/doi/10.1073/pnas.0900943106.

Vinod, H. D. (2010), "Superior Estimation and Inference Avoiding Heteroscedasticity and Flawed Pivots: R-example of Inflation Unemployment Trade-Off," in "Advances in Social Science Research Using R,", ed. Vinod, H. D., New York: Springer, pp. 39-63.

- (2015), "Generalized Correlation and Kernel Causality with Applications in Development Economics," Communications in Statistics - Simulation and Computation, accepted Nov. 10, 2015, URL Âăhttp://dx.doi.org/ 10.1080/03610918.2015.1122048.

World Bank (2013), "Working for World Free of Poverty," Tech. rep., IBRD, Washington DC, URL http://iresearch.worldbank.org/PovcalNet/ index.htm?1. 